\title{
Some Fundamental Results on Fuzzy Conformable Differential Calculus
}

\author{
Awais Younus ${ }^{a}$, Muhammad Asif $^{a}$, Usama AtTa $^{a}$, Tehmina BashiR $^{a}$, Thabet $^{\circ}$ \\ ABDELJAWAD ${ }^{\mathrm{b}, *}$ (1) \\ a Centre for Advanced Studies in Pure and Applied Mathematics, Bahauddin Zakaryia \\ University, Multan, Pakistan \\ b Department of Mathematics and General Sciences, Prince Sultan University, 11586 \\ Riyadh,Saudi Arabia
}

- Received: 30 August 2021 • Accepted: 01 November 2021

- Published Online: 30 December 2021

\begin{abstract}
In this paper, we combine fuzzy calculus, and conformable calculus to introduce the fuzzy conformable calculus. We define the fuzzy conformable derivative of order $2 \Psi$ and generalize it to derivatives of order $p \Psi$. Several properties on difference, product, sum, and addition of two fuzzy-valued functions are provided which are used in the solution of the fuzzy conformable differential equations. Also, examples in each case are given to illustrate the utility of our results.
\end{abstract}

Keywords: Fuzzy-valued functions, fractional derivative, strongly generalized conformable derivative. MSC: 26A33, 26E50.

\section{Introduction}

In the real world, the data sometimes cannot be collected precisely. For example, the water level of a river cannot be measured in an exact manner because of fluctuation. Similarly, the temperature in a room also cannot be measured precisely because of a similar reason. Therefore, the fuzzy numbers are used to deal with non-precise quantities possessing vagueness. Thus a more appropriate way to describe the water level is to take the water level " around 25 meters" as a fuzzy number.

The concept of the fuzzy sets was originally introduced by Zadeh as an extension of the classical set [1]. The basic arithmetic structure of the fuzzy numbers was later developed by Mizumoto and Tanaka [2], Dubois and Prade [3]. Fuzzy calculus is a basic structure in fuzzy mathematics. The concept of the fuzzy derivative was first introduced by Chang, and Zadeh ([4], [5]). H-derivative [6] was the starting point of the topic of 
the set differential equations, and later for the fuzzy differential equations. But this technique has the disadvantage that it does not exist for all the fuzzy-valued functions. Also, the solution obtained using H-derivative is not bounded.

Bede [7] introduced a generalization of $\mathrm{H}$-derivative based on the lateral type of derivatives called strongly generalized differentiability to remove this difficulty. Here derivative exists, and the solution of the fuzzy differential equations may have a decreasing length of support, but the uniqueness is lost. However, this disadvantage can be seen as an advantage since we can choose the singular points where the support of the solution changes its monotonicity. So we can obtain reversible solutions, stable, and almost periodic solutions, and asymptotic behavior of solutions of the fuzzy differential equations $[8,9,10,11]$.

Fractional calculus is a generalization of ordinary calculus where derivatives and integrals of arbitrary order are defined. By considering fractional derivatives, we have infinite choices of derivative orders that we can consider, and with it, we can determine what is the order that better describes the dynamics of the model. So we can model our problem more effectively.

The history of fractional calculus is the same as the history of ordinary calculus. In the answer to the renowned mathematician L' Hospital's question, if $\frac{d^{m}}{d v^{m}}$, then what does it mean if $m=1 / 2$, different explanations of the fractional derivative are presented. The most famous approaches to fractional derivatives are Caputo derivative, Letnikove, and R-L derivative $[12,13]$. Few properties of these fractional-order derivatives are similar to the classical order derivatives. However, there are a few complications.

1. These definitions, however, are non-local, which makes them unsuitable for investigating properties related to local scaling or fractional differentiability.

2. Riemann Liouville's derivative does not fulfill $\mathrm{D}^{\Psi}(1)=0$.

3. For Caputo's derivative, we have to assume that the function is differentiable. Otherwise, we cannot apply this definition.

In short, all fractional derivatives are deficient in some mathematical properties like product rule, chain rule, and quotient rule. Therefore, the solution of differential equations is not easy to obtain using these definitions.

Recently, [14] introduced a new definition of fractional derivative called conformable derivative. This new definition is different from other fractional derivatives and similar to the classical definition of the derivative. It depends on the limit definition of the derivative of a function. So this definition seems to be a natural extension of the ordinary derivative. [15] defined left and right conformable derivative, conformable power series, and conformable Laplace transform. This theory has attracted many researchers to work within and so many new concepts are introduced in conformable fractional calculus. For more research works, we refer the interested reader to ([16], [17], [18]) and the references therein. Modeling real-life problems with conformable derivatives ([19], [20]). Other fractional derivatives do not have geometrical interpretation but conformable derivative has [21]. For relevant concepts on conformable calculus, see for example ([22], [23], [24]).

A combination of these theories would be a ground-breaking strategy and can model better real-world phenomena. Recently, few attempts have been made in this area such as [25]. The authors introduced a fuzzy conformable derivative of order $\Psi$. Now, we 
have extended their work to the fuzzy conformable derivative of order $2 \Psi$, and also provide a generalization to the fuzzy conformable derivative of order $p \Psi$. Also, we have proved relevant results on the fuzzy conformable derivative of addition, subtraction, multiplication, and H-difference of two the fuzzy-valued functions.

We organized this paper as follows: Section 2 includes the basic concepts on the fuzzy and conformable differential calculus. In Section 3, we have investigated the fuzzy conformable derivative of order $\Psi$, Section 4 contains the fuzzy conformable derivative of order $2 \Psi$ while in Section 5, we have provided the generalization of the fuzzy conformable derivative up to order $p \Psi$. In the end, a summary of our results is presented.

\section{Basic Concepts}

\subsection{Fuzzy Calculus}

Definition 2.1. A fuzzy set is a map $\eta: \mathbb{R} \rightarrow[0,1]$ which generalizes classical sets from $\{0,1\}$ to $[0,1]$.

Definition 2.2. ([26]) A fuzzy number $\eta$ is a fuzzy set that satisfies some additional properties of convexity, normality, upper semi-continuity, and compact support.

We use $\mathbb{R}_{\Phi}$ to denote the space of all the real fuzzy numbers.

Definition 2.3. For $0 \leqslant \gamma<1, \gamma$-cuts for a fuzzy number $\eta$ is defined as

$$
[\eta]^{\gamma}=\{v \in \mathbb{R}: \eta(v) \geqslant \gamma\} .
$$

Example 2.4. A triangular fuzzy number $\eta$, denoted by an ordered triple $(a, b, c)$, with the condition $a \leqslant b \leqslant c$. The $\gamma$-cuts associated with the triangular fuzzy number $\eta$ are

$$
\begin{aligned}
& \left(\eta_{*}\right)_{\gamma}=a+(b-a) \gamma, \\
& \left(\eta^{*}\right)_{\gamma}=c-(c-b) \gamma,
\end{aligned}
$$

where $[\eta]^{\gamma}=\left[\left(\eta_{*}\right)_{\gamma},\left(\eta^{*}\right)_{\gamma}\right]$.

Definition 2.5. ([27])The Hausdorff metric $D_{H}: \mathbb{R}_{\Phi} \times \mathbb{R}_{\Phi} \rightarrow \mathbb{R}_{0}^{+}$on the space of the fuzzy numbers is defined as

$$
D_{\mathrm{H}}(\eta, v)=\sup _{0<\gamma \leqslant 1} \max \left\{\left|\left(\eta_{*}\right)_{\gamma}-\left(v_{*}\right)_{\gamma}\right|,\left|\left(\eta^{*}\right)_{\gamma}-\left(v^{*}\right)_{\gamma}\right|\right\}
$$

$[\eta]^{\gamma}=\left[\left(\eta_{*}\right)_{\gamma},\left(\eta^{*}\right)_{\gamma}\right],[v]^{\gamma}=\left[\left(v_{*}\right)_{\gamma},\left(v^{*}\right)_{\gamma}\right]$.

Proposition 2.6. Arithmetic operations on the space of the fuzzy numbers are generalized from that of real intervals. If $\eta, v \in \mathbb{R}_{\Phi}$, with $\gamma$-cuts $[\eta]^{\gamma}=\left[\left(\eta_{*}\right)_{\gamma},\left(\eta^{*}\right)_{\gamma}\right],[v]^{\gamma}=\left[\left(v_{*}\right)_{\gamma},\left(v^{*}\right)_{\gamma}\right]$. Then addition on space of the fuzzy numbers by $\gamma$-cuts is defined as

$$
[\eta+v]^{\gamma}=\left[\left(\eta_{*}\right)_{\gamma}+\left(v_{*}\right)_{\gamma^{\prime}}\left(\eta^{*}\right)_{\gamma}+\left(v^{*}\right)_{\gamma}\right]
$$

Scalar multiplication is defined as

$$
[\alpha \odot \eta]^{\gamma}=\left\{\begin{array}{l}
{\left[\alpha \cdot\left(\eta_{*}\right)_{\gamma}, \alpha \cdot\left(\eta^{*}\right)_{\gamma}\right] \text { if } \alpha \in \mathbb{R}>0,} \\
{\left[\alpha \cdot\left(\eta^{*}\right)_{\gamma}, \alpha \cdot\left(\eta_{*}\right)_{\gamma}\right], \alpha \in \mathbb{R}<0 .}
\end{array}\right.
$$

Subtraction on the space of fuzzy numbers is $[(\eta)-(v)]^{\gamma}=\left[\left(\eta_{*}\right)_{\gamma}-\left(v^{*}\right)_{\gamma},\left(\eta^{*}\right)_{\gamma}-\left(v_{*}\right)_{\gamma}\right]$. 
Definition 2.7. A fuzzy-valued function $\Phi$ is a function from arbitrary interval to $\mathbb{R}_{\Phi}$. A fuzzy-valued function in $\gamma$-cuts form is represented by $[\Phi(v)]^{\gamma}=\left[\left(\Phi_{*}\right)_{\gamma}(v),\left(\Phi^{*}\right)_{\gamma}(v)\right]$.

Definition 2.8. Hukuhara derivative of a fuzzy-valued function $\Phi:(a, b) \rightarrow \mathbb{R}_{\Phi}$ is a fuzzy-valued function $\Phi^{\prime}$ such that for all $\theta>0(\exists) \Phi\left(v_{0}+\theta\right) \ominus \Phi\left(v_{0}\right), \Phi\left(v_{0}\right) \ominus \Phi\left(v_{0}-\theta\right)$

$$
\lim _{\theta \rightarrow 0} \frac{\Phi\left(v_{0}+\theta\right) \ominus \Phi\left(v_{0}\right)}{\theta}=\lim _{\theta \rightarrow 0} \frac{\Phi\left(v_{0}\right) \ominus \Phi\left(v_{0}-\theta\right)}{\theta} .
$$

Definition 2.9. ([7]) A fuzzy-valued function $\Phi$ is strongly generalized differentiable at $v_{0} \in(\mathrm{a}, \mathrm{b})$ if $(\exists) \Phi^{\prime}\left(v_{0}\right) \in \mathbb{R}_{\Phi}$ such that

1. $(\forall) \theta>0$, there exist $\mathrm{H}$-differences $\Phi\left(v_{0}+\theta\right) \ominus \Phi\left(v_{0}\right), \Phi\left(v_{0}\right) \ominus \Phi\left(v_{0}-\theta\right)$ such that

$$
\lim _{\theta \rightarrow 0} \frac{\Phi\left(v_{0}+\theta\right) \ominus \Phi\left(v_{0}\right)}{\theta}=\lim _{\theta \rightarrow 0} \frac{\Phi\left(v_{0}\right) \ominus \Phi\left(v_{0}-\theta\right)}{\theta} .
$$

2. $(\forall) \theta>0$, there exist H-differences $\Phi\left(v_{0}\right) \ominus \Phi\left(v_{0}+\theta\right), \Phi\left(v_{0}-\theta\right) \ominus \Phi\left(v_{0}\right)$ and we have

$$
\lim _{\theta \rightarrow 0} \frac{\Phi\left(v_{0}\right) \ominus \Phi\left(v_{0}+\theta\right)}{-\theta}=\lim _{\theta \rightarrow 0} \frac{\Phi\left(v_{0}-\theta\right) \ominus \Phi\left(v_{0}\right)}{-\theta} .
$$

Definition 2.10. We say that $\Phi$ is a strongly generalized differentiable of type (1) if $\Phi$ is a strongly generalized differentiable in the first case and a differentiable of type (2) if $\Phi$ is strongly generalized differentiable in the second case.

Definition 2.11. A fuzzy-valued function $\Phi$ is strongly generalized differentiable of order two if $(\exists)$ a fuzzy number $\Phi^{\prime \prime}(v)$ such that

1. $(\forall) \theta>0$, there exist H-differences $\Phi^{\prime}\left(v_{0}+\theta\right) \ominus \Phi^{\prime}(v)$ and $\Phi^{\prime}(v) \ominus \Phi^{\prime}(v-\theta)$ s.that

$$
\lim _{\theta \rightarrow 0} \frac{\Phi^{\prime}\left(v_{0}+\theta\right) \ominus \Phi^{\prime}\left(v_{0}\right)}{\theta}=\lim _{\theta \rightarrow 0} \frac{\Phi^{\prime}\left(v_{0}\right) \ominus \Phi^{\prime}\left(v_{0} \ominus \theta\right)}{\theta} .
$$

2. $(\forall) \theta>0$, there exist H-differences $\Phi^{\prime}\left(v_{0}\right) \ominus \Phi^{\prime}\left(v_{0}+\theta\right)$ and $\Phi^{\prime}\left(v_{0}-\theta\right) \ominus \Phi^{\prime}\left(v_{0}\right)$ such that

$$
\lim _{\theta \rightarrow 0} \frac{\Phi^{\prime}\left(v_{0}\right) \ominus \Phi^{\prime}\left(v_{0}+\theta\right)}{-\theta}=\lim _{\theta \rightarrow 0} \frac{\Phi^{\prime}\left(v_{0}-\theta\right) \ominus \Phi^{\prime}\left(v_{0}\right)}{-\theta} .
$$

1. If $\Phi\left(v_{0}\right)$ and $\Phi^{\prime}\left(v_{0}\right)$ both are differentiable of type (1) or type (2), then case (1) of the above definition can be written in the form

$$
\lim _{\theta \rightarrow 0} \frac{\Phi\left(v_{0}+2 \theta\right)+\Phi\left(v_{0}\right) \odot 2 \Phi\left(v_{0}+\theta\right)}{\theta^{2}} .
$$

2. If $\Phi\left(v_{0}\right)$ is differentiable of type (2) and $\Phi^{\prime}\left(v_{0}\right)$ is differentiable of type (1), then case (2) of the above definition can be written in the form

$$
\lim _{\theta \rightarrow 0} \frac{2 \Phi\left(v_{0}+\theta\right) \ominus\left(\Phi\left(v_{0}\right)\right)+\Phi\left(v_{0}+2 \theta\right)}{-\theta^{2}} .
$$


Definition 2.12. A fuzzy-valued function $\Phi$ is strongly generalized differentiable of order $p$ at $v_{0} \in(a, b)$ if $(\exists) \Phi^{(p)}\left(v_{0}\right) \in \mathbb{R}_{\Phi}$ such that

1. $(\forall) \theta>0$, there exist H-differences $\Phi^{(p-1)}\left(v_{0}+\theta\right) \ominus \Phi^{(p-1)}\left(v_{0}\right), \Phi^{(p-1)}\left(v_{0}\right) \ominus \Phi^{(p-1)}\left(v_{0} \ominus\right.$ $\theta)$ such that

$$
\lim _{\theta \rightarrow 0} \frac{\Phi^{(p-1)}\left(v_{0}+\theta\right) \ominus \Phi^{(p-1)}\left(v_{0}\right)}{\theta}=\lim _{\theta \rightarrow 0} \frac{\Phi^{(p-1)}\left(v_{0}\right) \ominus \Phi^{(p-1)}\left(v_{0} \ominus \theta\right)}{\theta} .
$$

2. $(\forall) \theta>0$, there exist H-differences $\Phi^{(p-1)}\left(v_{0}\right) \ominus \Phi^{(p-1)}\left(v_{0}+\theta\right), \Phi^{(p-1)}\left(v_{0}-\theta\right) \ominus$ $\Phi^{(p-1)}\left(v_{0}\right)$

$$
\lim _{\theta \rightarrow 0} \frac{\Phi^{(p-1)}\left(v_{0}\right) \ominus \Phi^{(p-1)}\left(v_{0}+\theta\right)}{-\theta}=\lim _{\theta \rightarrow 0} \frac{\Phi^{(p-1)}\left(v_{0}-\theta\right) \ominus \Phi^{(p-1)}\left(v_{0}\right)}{-\theta} .
$$

2.2. Conformable Calculus

Definition 2.13. Conformable derivative of order $\Psi$ of a non-negative real-valued function $\Phi$ is defined as

$$
\Phi^{\Psi}(v)=\lim _{\theta \rightarrow 0} \frac{\Phi\left(v+\theta v^{1-\Psi}\right)-\Phi(v)}{\theta}
$$

where $\Psi \in(0,1]$. If the conformable derivative of order $\Psi$ exists, then we say that $\Phi$ is $\Psi$-differentiable.

Remark 2.14. Note that the conformable derivative becomes equal to the usual derivative when $\Psi=1$.

Proposition 2.15. Relation between $\Psi$-differentiable function $\Phi$ and a function $\Phi$ differentiable with classical derivative is

$$
\Phi^{\Psi}(v)=v^{1-\Psi} \Phi(v) .
$$

Theorem 2.16. Taylor's series for an infinitely differentiable function $\Phi$ with respect to conformable derivative at a point $\mathrm{u}$ is defined as

$$
\Phi(v)=\sum_{p=0}^{\infty} \frac{u^{p-p \Psi}(v-a)^{p} \Phi^{p}(u)}{p !}
$$

Example 2.17. Maclaurin's series (Taylor series evaluated at 0 ) for $e^{\frac{v^{\Psi}}{\Psi}}$ and $e^{-\frac{v^{\Psi}}{\Psi}}$ has the form

$$
\begin{aligned}
e^{\frac{v^{\Psi}}{\Psi}} & =\sum_{\ell=0}^{\infty} \frac{v^{(2 \ell+1) \Psi}}{\ell ! \Psi 2 \ell+1} \\
e^{\frac{-v^{\Psi}}{\Psi}} & =\sum_{\ell=0}^{\infty} \frac{v^{(2 \ell+1) \Psi}}{\ell ! \Psi^{2 \ell+1}}
\end{aligned}
$$

Similar to the classical case, Maclaurin's series for $\cos v$ is

$$
\frac{e^{\frac{v^{\Psi}}{\Psi}}+e^{\frac{-\nu^{\Psi}}{\Psi}}}{2}=\cosh \nu=\sum_{\ell=0}^{\infty} \frac{v^{(2 \ell) \Psi}}{\Psi^{2 \ell}(2 \ell) !}
$$


Also for $\sin v$, we have

$$
\frac{e^{\frac{v^{\Psi}}{\Psi}}-e^{\frac{-v^{\Psi}}{\Psi}}}{2}=\sinh v=\sum_{\ell=0}^{\infty} \frac{v^{(2 \ell+1) \Psi}}{\Psi^{2 \ell+1}(2 \ell+1) !}
$$

\section{Fuzzy Conformable Derivative of Order $\Psi$}

Definition 3.1. A fuzzy-valued function $\Phi$ is called conformable H-differentiable of order $\Psi$ at a point $v \in(a, b)$ if

$(\forall) \theta>0$, H-differences $\Phi\left(v+\theta v^{1-\Psi}\right) \odot \Phi(v)$, and $\Phi(v) \odot \Phi\left(v-\theta v^{1-\Psi}\right)$ exist, and we have

$$
\lim _{\theta \rightarrow 0} \frac{\Phi\left(v+\theta v^{1-\Psi}\right) \ominus \Phi(v)}{\theta}=\lim _{\theta \rightarrow 0} \frac{\Phi(v) \ominus \Phi\left(v-\theta v^{1-\Psi}\right)}{\theta} .
$$

Note that equation (3.1) has the form

$$
\lim _{\theta \rightarrow 0} \frac{\Phi\left(v+\theta v^{\lceil\Psi\rceil-\Psi}\right) \ominus \Phi(v)}{\theta}=\lim _{\theta \rightarrow 0} \frac{\Phi(v) \ominus \Phi\left(v-\theta v^{\lceil\Psi\rceil-\Psi}\right)}{\theta},
$$

this can also be written as

$$
\Phi^{\Psi}(v)=v^{\lceil\Psi\rceil-\Psi} \Phi^{\prime}(v),
$$

where $\lceil\cdot\rceil$ is the ceiling function.

Definition 3.2. Let $\Phi$ be a fuzzy-valued function having a strongly generalized conformable derivative of order $p \Psi$. Then the strongly generalized conformable derivative of $\Phi$ of order $\Psi$ is defined as

$\Phi^{\Psi}(v)=\lim _{\theta \rightarrow 0} \frac{\Phi^{\lceil\Psi\rceil-1}\left(v+\theta v^{\lceil\Psi\rceil-\Psi}\right) \ominus \Phi^{\lceil\Psi\rceil-1}(v)}{\theta}=\lim _{\theta \rightarrow 0} \frac{\Phi^{\lceil\Psi\rceil-1}(v) \ominus \Phi^{\lceil\Psi\rceil-1}\left(v-\theta v^{\lceil\Psi\urcorner-\Psi}\right)}{\theta}$,

where $\Psi \in(p, p+1]$, and [.] is the ceiling function.

Lemma 3.3. For a fuzzy-valued function $\Phi$, which is strongly generalized conformable differentiable of order $\Psi$, the following expression holds with $\Psi \in(p, p+1]$

$$
\Phi^{\Psi}(v)=v^{p-\Psi} \Phi^{p}(v) .
$$

Proof.

$$
\begin{aligned}
\Phi^{\Psi}(v) & =\lim _{\theta \rightarrow 0} \frac{\Phi^{\lceil\Psi\rceil-1}\left(v+\theta v^{\lceil\Psi\rceil-\Psi}\right)-\Phi^{\lceil\Psi\rceil-1}(v)}{\Phi^{\lceil\Psi\rceil-1}\left(v+\theta v^{\lceil\Psi}-\Psi\right)-\Phi^{\lceil\Psi\rceil-1}(v)} \\
& =\lim _{\theta \rightarrow 0} \frac{\Phi^{\lceil\Psi\rceil-\Psi}}{\theta v^{\lceil\Psi]-\Psi}} \\
& =v^{p-\Psi} \Phi^{p}(\nu) .
\end{aligned}
$$

Remark 3.4. For $\Psi=1$, conformable $\mathrm{H}$-derivative coincides with the first-order $\mathrm{H}$ derivative. However, the zero-order conformable H-derivative $\Phi^{0}(v)$ of a fuzzy-valued function does not return the fuzzy-valued function $\Phi(v)$, because

$$
\Phi^{0}(v)=\mathrm{t} \cdot \Phi(v) \neq \Phi(v) .
$$


Proposition 3.5. For a fuzzy-valued function $\Phi$ with $\gamma$-cuts $[\Phi(v)]^{\gamma}=\left[\left(\Phi_{*}\right)_{\gamma}(v),\left(\Phi^{*}\right)_{\gamma}(v)\right]$ if $\left(\Phi_{*}\right)_{\gamma}$ and $\left(\Phi^{*}\right)_{\gamma}$ are conformable differentiable, then $[\Phi(v)]^{\gamma}$ is also conformable $\mathrm{H}$-differentiable and we have

$$
\left[\Phi^{\Psi}(v)\right]^{\gamma}=\left[\left(\Phi_{*}\right)_{\gamma}^{\Psi}(v),\left(\Phi^{*}\right)_{\gamma}^{\Psi}(v)\right],
$$

Proof. By definition of H-conformable differentiability, we have

$$
\left[\Phi^{\Psi}(v)\right]^{\gamma}=\left[\lim _{\theta \rightarrow 0} \frac{\Phi\left(v+\theta v^{1-\Psi}\right) \ominus \Phi(v)}{\theta}\right]^{\gamma} .
$$

Using the definition of $\mathrm{H}$-conformable differentiability, and parametric form of level sets, we obtain

$$
\left[\Phi^{\Psi}(v)\right]^{\gamma}=\left[\lim _{\theta \rightarrow 0} \frac{\Phi_{*}\left(v+\theta v^{1-\Psi}\right)-\Phi_{*}(v), \Phi^{*}\left(v+\theta v^{1-\Psi}\right)-\Phi^{*}(v)}{\theta}\right]^{\gamma} .
$$

This gives us our result in the form

$$
\left[\Phi^{\Psi}(v)\right]^{\gamma}=\left[\lim _{\theta \rightarrow 0} \frac{\Phi_{*}\left(v+\theta v^{1-\Psi}\right)-\Phi_{*}(v)}{\theta}, \frac{\Phi^{*}\left(v+\theta v^{1-\Psi}\right)-\Phi^{*}(v)}{\theta}\right]^{\gamma} .
$$

Thus proved.

Remark 3.6. But the converse of the above proposition is not true. i.e. The H-conformable differentiability of $\Phi$ does not guarantee the existence of conformable differentiability of $\Phi_{*}$ and $\Phi^{*}$. For example, a fuzzy-valued function $\Phi(v)=\sin \Phi(v) \odot(-1,0,1)$ for $v \in[0, \pi]$ with $\gamma$-cuts $[\Phi(v)]^{\gamma}=\sin t \cdot[(1+\gamma),(3-\gamma)]$. Then, $\Phi(v)$ is conformable Hdifferentiable, but the level-sets are not conformable differentiable for $\left[\frac{\pi}{2}, \pi\right]$.

Remark 3.7. A conformable H-differentiable function $\Phi$ may not be differentiable with the $\mathrm{H}$-derivative.

For example, for $\Phi(v)=2 v^{\frac{1}{2}} \odot(-1,0,1)$, we obtain $\Phi^{\frac{1}{2}}(v)=1 \odot(-1,0,1)$. Thus we have

$$
\Phi^{\frac{1}{2}}(0)=\lim _{v \rightarrow 0} \Phi^{\frac{1}{2}}(v)=1 \odot(-1,0,1) .
$$

So $\Phi(v)$ is conformable $\mathrm{H}$-differentiable at 0 , but its $\mathrm{H}$-derivative does not exist.

Remark 3.8. Conformable H-derivative of a fuzzy-valued function has some disadvantages such as

1. It does not gives a bounded solution.

2. For a fuzzy-valued function $\Phi(v)=\eta \odot \psi(v)$, where $\eta$ is a fuzzy number and $\psi$ is a real-valued conformable differentiable function, conformable $\mathrm{H}$-derivative does not exist when $\psi^{\Psi}(v)<0$. So we use strongly generalized conformable differentiability which is a generalization of conformable H-differentiability.

Definition 3.9. ([25]) A fuzzy-valued function $\Phi$ is called strongly generalized $\Psi$ - differentiable at a point $v \in(a, b)$ if 
1. $(\forall) \theta>0$, H-differences $\Phi\left(v+\theta v^{1-\Psi}\right) \ominus \Phi(v)$ and $\Phi(v) \ominus \Phi\left(v-\theta v^{1-\Psi}\right)$ exist, and we have

$$
\lim _{\theta \rightarrow 0} \frac{\Phi\left(v+\theta v^{1-\Psi}\right) \ominus \Phi(v)}{\theta}=\lim _{\theta \rightarrow 0} \frac{\Phi(v) \odot \Phi\left(v-\theta v^{1-\Psi}\right)}{\theta} .
$$

2. $(\forall) \theta>0$, H-differences $\Phi(v) \odot \Phi\left(v+\theta v^{1-\Psi}\right)$, and $\Phi\left(v-\theta v^{1-\Psi}\right) \ominus \Phi(v)$ exist, and we have

$$
\lim _{\theta \rightarrow 0} \frac{\Phi(v) \ominus \Phi\left(v+\theta v^{1-\Psi}\right)}{-\theta}=\lim _{\theta \rightarrow 0} \frac{\Phi\left(v-\theta v^{1-\Psi}\right) \ominus \Phi(v)}{-\theta} .
$$

If a strongly generalized $\Psi$-derivative of a fuzzy-valued function $\Phi$ exists, then we say that $\Phi$ is strongly generalized $\Psi$-differentiable on $(a, b)$.

Definition 3.10. We say that $\Phi$ is strongly generalized differentiable of type ( $\Psi-1)$ if $\Phi$ is differentiable in the first case, and $\Phi$ is strongly generalized differentiable of type ( $\Psi-2)$ if $\Phi$ is differentiable in the second case.

Example 3.11. Define a fuzzy-valued function $\Phi$ as

$$
\Phi(v)=\eta \odot \psi(v),
$$

where $\eta$ is a fuzzy number, and $\psi:(0, \infty) \rightarrow \mathbb{R}$ is $\psi$-differentiable real-valued function. If $\psi^{\Psi}(v)>0$, then $\Phi$ is differentiable of type ( $\left.\Psi-1\right)$, and if $\psi^{\Psi}(v)<0$, then $\Phi$ is differentiable of type $(\Psi-2)$.

Theorem 3.12. Let $\Phi:(a, b) \rightarrow \mathbb{R}_{\Phi}$, then $\gamma$-cuts representation of $\Phi$ is

$$
[\Phi(v)]^{\gamma}=\left[\left(\Phi_{*}\right)_{\gamma}(\nu),\left(\Phi^{*}\right)_{\gamma}(v)\right]
$$

1. If $\Phi$ is differentiable of type ( $\Psi-1)$, then $\Phi_{*}$ and $\Phi^{*}$ are $\Psi$-differentiable, and we get

$$
\left[\Phi^{\Psi}(v)\right]^{\gamma}=\left[\left(\Phi_{*}\right)_{\gamma}^{\Psi}(v),\left(\Phi^{*}\right)_{\gamma}^{\Psi}(v)\right] .
$$

2. If $\Phi$ is differentiable of type $(\Psi-2)$, then $\Phi^{*}$ and $\Phi_{*}$ are $\Psi$ - differentiable, and we get

$$
\left[\Phi^{\Psi}(v)\right]^{\gamma}=\left[\left(\Phi^{*}\right)_{\gamma}^{\Psi}(v),\left(\Phi_{*}\right)_{\gamma}^{\Psi}(v)\right] .
$$

Proof. We prove the case (1) only. Case (2) can be proved similarly.

Since $\Phi$ is differentiable of type $(\Psi-1)$, therefore we have

$$
\Phi\left(v+\theta v^{1-\Psi}\right) \ominus \Phi(v)=\left[\Phi_{*}\left(v+\theta v^{1-\Psi}\right)-\Phi_{*}(v), \Phi^{*}\left(v+\theta v^{1-\Psi}\right)-\Phi^{*}(v)\right] .
$$

Similarly,

$$
\Phi(v) \odot \Phi\left(v-\theta v^{1-\Psi}\right)=\left[\Phi_{*}(v)-\Phi_{*}\left(v-\theta v^{1-\Psi}\right), \Phi^{*}(v)-\Phi^{*}\left(v-\theta v^{1-\Psi}\right)\right] .
$$

Now by dividing both sides by $\frac{1}{\theta}$, and taking $\lim _{\theta \rightarrow 0}$ on both sides of equation (3.5), and equation (3.6), we obtain our required result. 
Theorem 3.13. Relation between $\Psi$-strongly generalized differentiable fuzzy-valued function and strongly generalized differentiable fuzzy-valued function is

1. If $\Phi$ is strongly generalized differentiable of type ( $\Psi-1)$, then we have

$$
\Phi_{(1)}^{(\Psi)}(v)=v^{1-\Psi} \Phi_{(1)}^{\prime}(v)
$$

2. If $\Phi$ is strongly generalized differentiable of type ( $\Psi-2)$, then we have

$$
\Phi^{(\Psi-2)}(v)=v^{1-\Psi} \Phi_{(2)}^{\prime}(v) .
$$

Proof. Proof for case (2) is provided here. Case (1) can be easily proved similarly.

$$
\left[\Phi^{(\Psi-2)}(v)\right]^{\gamma}=\left[\lim _{\theta \rightarrow 0^{-}} \frac{\left(\Phi^{*}\right)_{\gamma}(v)-\left(\left(\Phi^{*}\right)_{\gamma}\left(v+\theta v^{1-\Psi}\right)\right)}{\theta}, \lim _{\theta \rightarrow 0^{-}} \frac{\left(\Phi_{*}\right)_{\gamma}(v)-\left(\left(\Phi_{*}\right)_{\gamma}\left(v+\theta v^{1-\Psi}\right)\right)}{\theta}\right] .
$$

Put $\theta v^{1-\Psi}=\lambda$, we have the above expression in the form

$$
\left[\Phi^{(\Psi-2)}(v)\right]^{\gamma}=\left[\lim _{\lambda \rightarrow 0} \frac{\left(\Phi^{*}\right)_{\gamma}(v)-\left(\left(\Phi^{*}\right)_{\gamma}(v+\lambda)\right)}{\lambda v^{\Psi-1}}, \lim _{\lambda \rightarrow 0} \frac{\left(\Phi_{*}\right)_{\gamma}(v)-\left(\left(\Phi_{*}\right)_{\gamma}(v+\lambda)\right)}{\lambda v^{\Psi-1}}\right] .
$$

So we have

$$
\begin{aligned}
& {\left[\Phi^{(\Psi-2)}(v)\right]^{\gamma}=\left[v^{1-\Psi} \lim _{\lambda \rightarrow 0} \frac{\left(\Phi^{*}\right)_{\gamma}(v)-\left(\left(\Phi^{*}\right)_{\gamma}(v+\lambda)\right)}{\lambda}, \lim _{\lambda \rightarrow 0} v^{1-\Psi} \frac{\left(\Phi_{*}\right)_{\gamma}(v)-\left(\left(\Phi_{*}\right)_{\gamma}(v+\lambda)\right)}{\lambda}\right],} \\
& {\left[\Phi^{(\Psi-2)}(v)\right]^{\gamma}=v^{1-\Psi}\left[\lim _{\lambda \rightarrow 0} \frac{\left(\Phi^{*}\right)_{\gamma}(v)-\left(\left(\Phi^{*}\right)_{\gamma}(v+\lambda)\right)}{\lambda}, \lim _{\lambda \rightarrow 0} \frac{\left(\Phi_{*}\right)_{\gamma}(v)-\left(\left(\Phi_{*}\right)_{\gamma}(v+\lambda)\right)}{\lambda}\right] .}
\end{aligned}
$$

Thus our required result is obtained.

$$
\left[\Phi^{(\Psi-2)}(v)\right]^{\gamma}=v^{1-\Psi}\left[\Phi_{(2)}^{\prime}(v)\right] .
$$

Theorem 3.14. If a fuzzy-valued function $\Phi$ is $\Psi$-strongly generalized differentiable at any point $v$, then $\Phi$ is continuous at $v$.

Proof. The proof is straight-forward so left for the reader.

1. Strongly generalized conformable derivative for fuzzy-valued function $\Phi$ does not obey index law for any non-negative constants $\Psi, \Delta \in(0,1)$, that is

$$
\Phi^{\Psi+\Delta}(v) \neq \Phi^{\Psi}(v) \Phi^{\Delta}(v) .
$$

Let $\Phi(v)=v^{2} \odot(-1,0,1), \Psi=1 / 2, \Delta=1 / 3$. Then

$$
\begin{array}{ll}
\Phi^{\frac{5}{6}}(v) & =2 v^{\frac{7}{6}} \odot(-1,0,1) \\
\Phi^{\frac{1}{3}}(v) \Phi^{\frac{1}{2}}(v) & =\frac{10}{3} v^{\frac{7}{6}} \odot(-1,0,1)
\end{array}
$$

However, this deficiency can be removed by taking $\Delta=1$. 
2. Strongly generalized conformable derivative for fuzzy-valued function $\Phi$ does not obey commutative law for any non-negative constants $\Psi, \Delta \in(0,1)$, that is

$$
\Phi^{\Delta}(v) \odot \Phi^{\Psi}(v) \neq \Phi^{\Psi}(v) \odot \Phi^{\Delta}(v) .
$$

This result also holds with $p \geqslant 2$.

3. Triangular inequality for strongly generalized conformable derivative also does not hold.

Lemma 3.15. ([29]) Let $\Phi$, and $\psi:(0, \infty) \rightarrow \mathbb{R}_{\Phi}$ have strongly generalized $\Psi$-differentiability of the same case at any point $\nu>0$, then $\Phi+\psi$ is strongly generalized $\Psi$-differentiable, and we have

$$
(\Phi+\psi)^{\Psi}(v)=\Phi^{\Psi}(v)+\psi^{\Psi}(v) .
$$

Theorem 3.16. Consider fuzzy-valued functions $\Phi$, and $\psi$ are strongly generalized differentiable of a different type. If $\Phi(v) \ominus \psi(v)$ exists, then $\Phi \ominus \psi$ is strongly generalized differentiable, and its derivative is

$$
(\Phi \ominus \psi)^{\Psi}(v)=\Phi^{\Psi}(v)+(-1) \psi^{\Psi}(v) .
$$

Proof. We take the case when $\Phi$ is differentiable of type (1), and $\psi$ is differentiable of type (2). Then there exist fuzzy numbers $\eta_{1}$, and $\eta_{2}$ such that

$$
\begin{aligned}
\Phi\left(v+\theta v^{1-\Psi}\right) & =\Phi(v)+\eta_{1}(v, \theta, \Psi), \\
\psi(v) & =\psi\left(v+\theta v^{1-\Psi}\right)+\eta_{2}(v, \theta, \Psi),
\end{aligned}
$$

and we get

$$
\Phi\left(v+\theta v^{1-\Psi}\right)+\psi(v)=\Phi(v)+\psi\left(v+\theta v^{1-\Psi}\right)+\eta_{1}(v, \theta, \Psi)+\eta_{2}(v, \theta, \Psi) .
$$

As H-difference $\Phi(v) \odot \psi(v)$, and $\Phi\left(v+\theta v^{1-\Psi}\right) \ominus \psi\left(v+\theta v^{1-\Psi}\right)$ exists, we get

$$
\left.\Phi(v)+\theta v^{1-\Psi}\right) \ominus \psi\left(v+\theta v^{1-\Psi}\right)=\Phi(v) \ominus \psi(v)+\eta_{1}(v, \theta, \Psi)+\eta_{2}(v, \theta, \Psi) .
$$

Since H-difference $\Phi\left(v+\theta v^{1-\Psi}\right) \ominus \psi\left(v+\theta v^{1-\Psi}\right) \ominus \Phi(v) \ominus \psi(v)$ exists, and we have

$$
\Phi\left(v+\theta v^{1-\Psi}\right) \ominus \psi\left(v+\theta v^{1-\Psi}\right) \ominus\left(\Phi(v) \ominus \psi(v)=\eta_{1}(v, \theta, \Psi)+\eta_{2}(v, \theta, \Psi) .\right.
$$

Similarly, we have that $(\exists) v_{1}(\nu, \theta, \Psi)$, and $v_{2}(v, \theta, \Psi)$ such that

$$
\begin{aligned}
\Phi(v) & =\Phi\left(v-\theta v^{1-\Psi}\right)+v_{1}(v, \theta, \Psi), \\
\psi\left(v-\theta v^{1-\Psi}\right) & =\psi(v)+v_{2}(v, \theta, \Psi) .
\end{aligned}
$$

So we have,

$$
\Phi(v) \ominus \psi(v) \odot \Phi\left(v-\theta v^{1-\Psi}\right) \ominus \psi\left(v-\theta v^{1-\Psi}\right)=v_{1}(v, \theta, \Psi)+v_{2}(v, \theta, \Psi) .
$$

Hence we obtain

$$
\lim _{\theta \rightarrow 0} \frac{\eta_{1}(\nu, \theta, \Psi)}{\theta}=\lim _{\theta \rightarrow 0} \frac{\eta_{2}(\nu, \theta, \Psi)}{\theta}=\Phi^{\Psi}(\nu) .
$$


Also

$$
\lim _{\theta \rightarrow 0} \frac{v_{1}(\nu, \theta, \Psi)}{\theta}=\lim _{\theta \rightarrow 0} \frac{v_{2}(\nu, \theta, \Psi)}{\theta}=(-1) \psi^{\Psi}(v) .
$$

So we have our required result.

$$
(\Phi \ominus \psi)^{\Psi}(v)=\Phi^{\Psi}(v)+(-1) \psi^{\Psi}(v) .
$$

Theorem 3.17. If $\Phi$ is $\Psi$-differentiable real-valued function, and $\psi$ is strongly generalized $\Psi$ differentiable fuzzy-valued function, then we have the following possibilities.

1 If $\Phi(v) \cdot \Phi^{\Psi}(v)>0$, and $\psi$ is differentiable of type $(\Psi-1)$, then $\Phi \odot \psi$ is differentiable of type $(\Psi-1)$, and

$$
(\Phi \odot \psi)^{\Psi}(v)=\Phi^{\Psi}(v) \odot \psi(v)+\Phi(v) \odot \psi^{\Psi}(v) .
$$

2 If $\Phi(v) \cdot \Phi^{\Psi}(v)<0$, and $\psi$ is differentiable of type $(\Psi-2)$, then $\Phi \odot \psi$ is differentiable of type $(\Psi-2)$, and

$$
(\Phi \odot \psi)^{\Psi}(v)=\Phi^{\Psi}(v) \odot \psi(v)+\Phi(v) \odot \psi^{\Psi}(v) .
$$

3 If $\Phi(v) \cdot \Phi^{\Psi}(v)>0, \psi$ is differentiable of type $(\Psi-2)$, and $\Phi \odot \psi$ satisfies condition (A), then $\Phi \odot \psi$ is differentiable, and

$$
(\Phi \odot \psi)^{\Psi}(v)=\Phi^{\Psi}(v) \odot \psi(v) \odot\left(-\Phi(v) \odot \psi^{\Psi}(v)\right) .
$$

4 If $\Phi(v) \cdot \Phi^{\Psi}(v)>0, \psi$ is differentiable of type ( $\left.\Psi-2\right)$, and $\Phi \odot \psi$ satisfies condition (B), then $\Phi \odot \psi$ is also differentiable, and we have

$$
(\Phi \odot \psi)^{\Psi}(v)=\Phi(v) \odot \psi^{\Psi}(v) \odot\left(-\Phi^{\Psi}(v) \odot \psi(v)\right) .
$$

5 If $\Phi(v) \cdot \Phi^{\Psi}(v)<0, \psi$ is differentiable of type $(\Psi-1)$, and $\Phi \odot \psi$ satisfies the condition (A), then $\Phi \odot \psi$ is also differentiable, and

$$
(\Phi \odot \psi)^{\Psi}(v)=\Phi(v) \odot \psi^{\Psi}(v) \odot\left(-\Phi^{\Psi}(v) \odot \psi(v)\right) .
$$

6 If $\Phi(v) \cdot \Phi^{\Psi}(v)<0, \psi$ is differentiable of type $(\Psi-2)$, and $\Phi \odot \psi$ satisfies the condition (B), then $\Phi \odot \psi$ is also differentiable, and we have

$$
(\Phi \odot \psi)^{\Psi}(v)=\Phi^{\Psi}(v) \odot \psi(v) \odot\left(-\Phi(v) \psi^{\Psi}(v)\right) .
$$

Proof. We provide proof of case (1) only, other cases can be proved in a similar pattern.

As $\Phi$ is a continuous fuzzy-valued function, therefore we have $\Phi(v), \Phi\left(v-\theta v^{1-\Psi}\right)$, $\Phi\left(v+\theta v^{1-\Psi}\right)$ with the same sign. As $\psi$ is differentiable of type $(\Psi-2)$, so there exists a fuzzy number $\eta_{1}(v, \theta, \Psi)$, and $v_{1}(v, \theta, \Psi)$ such that

$$
\psi(v)=\psi\left(v+\theta v^{1-\Psi}\right)+\eta_{1}(v, \theta, \Psi) .
$$


Also

$$
\Phi(v)=\Phi\left(v+\theta v^{1-\Psi}\right)+v_{1}(v, \theta, \Psi)
$$

Now we have

$$
\begin{gathered}
\Phi(v) \odot \psi(v)=\Phi\left(v+\theta v^{1-\Psi}\right) \odot \psi\left(v+\theta v^{1-\Psi}\right)+\Phi\left(v+\theta v^{1-\Psi}\right) \odot \eta_{1}(v, \theta, \Psi) \\
+v_{1}(v, \theta, \Psi) \odot \psi\left(v+\theta v^{1-\Psi}\right)+\eta_{1}(v, \theta, \Psi) \odot v_{1}(v, \theta, \Psi) .
\end{gathered}
$$

Since H-difference $\Phi(v) \odot \psi(v) \odot \Phi\left(v+\theta v^{1-\Psi}\right) \odot \psi\left(v+\theta v^{1-\Psi}\right)$ exists, so we get

$$
\begin{aligned}
& \Phi(v) \odot \psi(v) \odot \Phi\left(v+\theta v^{1-\Psi}\right) \odot \psi\left(v+\theta v^{1-\Psi}\right) \\
= & \Phi\left(v+\theta v^{1-\Psi}\right) \odot \eta_{1}(v, \theta, \Psi) \odot v_{1}(v, \theta, \Psi) \odot \psi\left(v+\theta v^{1-\Psi}\right) \\
& \odot \eta_{1}(v, \theta, \Psi) \odot v_{1}(v, \theta, \Psi) .
\end{aligned}
$$

Now, multiply both sides with $\frac{-1}{\theta}$, and taking $\lim _{\theta \rightarrow 0}$, we obtain our required result.

$$
\begin{aligned}
& \lim _{\theta \rightarrow 0} \frac{\Phi(v) \odot \psi(v) \odot \Phi\left(v+\theta v^{1-\Psi}\right) \odot \psi\left(v+\theta v^{1-\Psi}\right)}{-\theta} \\
= & \Phi^{\Psi}(v) \odot \psi(v)+\Phi(v) \odot \psi^{\Psi}(v) .
\end{aligned}
$$

Similarly, we obtain

$$
\begin{aligned}
& \lim _{\theta \rightarrow 0} \frac{\Phi\left(v-\theta v^{1-\Psi}\right) \odot \psi\left(v-\theta v^{1-\Psi}\right) \odot \Phi(v) \odot \psi(v)}{-\theta} \\
= & \Phi^{\Psi}(v) \odot \psi(v)+\Phi(v) \odot \psi^{\Psi}(v) .
\end{aligned}
$$

So our required result is obtained.

Theorem 3.18. Define a fuzzy-valued function by

$$
\Phi(v)=\eta \odot \psi(v),
$$

where $\eta$ is a fuzzy number, and if $\psi$ is differentiable, then $\Phi$ is also differentiable, and its fuzzy conformable derivative is given as

$$
\Phi^{\Psi}(v)=\eta \odot \psi^{\Psi}(v)
$$

If $\psi(v) \cdot \psi^{\Psi}(v)>0$, then $\Phi$ is differentiable of type ( $\left.\Psi-1\right)$.If $\psi(v) \cdot \psi^{\Psi}(v)<0$, then $\Phi$ is differentiable of type $(\Psi-2)$ and if $\psi(v) \cdot \psi^{\Psi}(v)=0$, then we have

$$
\Phi^{\Psi}(v)=\eta \odot \psi^{\Psi}(v) .
$$

Example 3.19. Define a fuzzy-valued function by

$$
\Phi(v)=\eta \odot \psi(v),
$$

where $\eta$ is a fuzzy number, $\psi(v)$ is a $\left(1, \frac{1}{2}\right)$ polynomial given by

$$
\psi(v)=v-v^{\frac{1}{2}}+1
$$

and $v \in(0,4)$. 
We have three possibilities.

Case 1: As $\psi(v) \cdot \psi^{\Psi}(v)>0$ for $v \in\left(\frac{5}{2}, 4\right)$, so by applying the above theorem, $\Phi$ is differentiable of type $(\Psi-1)$, and we have

$$
\Phi^{\Psi}(v)=\eta \odot \psi^{\Psi}(v)=\eta \odot\left(v^{\frac{1}{2}}-\frac{1}{2}\right) .
$$

Case 2: Since $\psi(v) \cdot \psi^{\Psi}(v)<0$ for $v \in(0,2.2)$, so by applying the above theorem, $\Phi$ is differentiable of type ( $\Psi-2)$, and we have

$$
\Phi^{\Psi}(v)=\eta \odot \psi^{\Psi}(v)=\eta \odot\left(v^{\frac{1}{2}}-\frac{1}{2}\right) .
$$

Case 3: We have $\psi(v) \cdot \psi^{\Psi}(v)=0$ for $(v)=2.25$, so by applying the above theorem, we have

$$
\Phi^{\Psi}(v)=\eta \odot \psi^{\Psi}(v)=\eta \odot\left(v^{\frac{1}{2}}-\frac{1}{2}\right) .
$$

Theorem 3.20. Let $\Phi$, and $\Psi$ are generalized differentiable fuzzy-valued functions at any $v \in$ $(0, \infty)$.

1 If $\Phi$ is differentiable of type ( $\Psi-1)$, and $\psi$ is differentiable of type $(\Psi-2)$, and $\Phi+\psi$ satisfies the condition (B), then $\Phi+\psi$ is also differentiable of type ( $\Psi-1)$, and we have

$$
(\Phi+\psi)^{\Psi}(v)=\psi^{\Psi}(v) \odot(-1) \Phi^{\Psi}(v) .
$$

2 If $\Phi$ is differentiable of type ( $\Psi-1)$, and $\psi$ is differentiable of type ( $\Psi-2)$, and $\Phi+\psi$ satisfies the condition (A), then $\Phi+\psi$ is also differentiable of type ( $\Psi-2)$, and we have

$$
(\Phi+\psi)^{\Psi}(v)=\Phi^{\Psi}(v) \odot(-1) \psi^{\Psi}(v) .
$$

Proof. We proof only case (1). Case (2) can be similarly proved.

Given that $\Phi$ is differentiable of type $(\Psi-1)$, so the H-differences $\Phi\left(v+\theta v^{1-\Psi}\right) \odot$ $\Phi(v)$, and $\Phi(v) \ominus \Phi\left(v-\theta v^{1-\Psi}\right)$ exist. So there exist fuzzy numbers $\eta_{1}(v, \theta, \Psi)$, and $\eta_{2}(v, \theta, \Psi)$ such that

$$
\Phi\left(v+\theta v^{1-\Psi}\right)=\Phi(v)+\eta_{1}(v, \theta, \Psi) .
$$

Also

$$
\Phi(v)=\Phi\left(v-\theta v^{1-\Psi}\right)+\eta_{2}(v, \theta, \Psi) .
$$

Since $\psi$ is differentiable of type $(\Psi-2)$. So there exist fuzzy numbers $v_{1}(v, \theta, \Psi)$, and $v_{2}(v, \theta, \Psi)$ such that

$$
\begin{aligned}
& \psi(v)=\psi\left(v+\theta v^{1-\Psi-}\right)+v_{1}(v, \theta, \Psi) . \\
& \psi\left(v-\theta v^{1-\Psi}\right)=\psi(v)+v_{2}(v, \theta, \Psi) .
\end{aligned}
$$

Also $(\Phi+\psi)$ satisfies the condition (B), then the H-differences $\psi(v)+\Phi(v) \ominus\left(\psi\left(v+\theta v^{1-\Psi}\right)+\right.$ $\left.\Phi v+\theta v^{1-\Psi}\right)$, and $\psi\left(v-\theta v^{1-\Psi}\right)+\Phi\left(v-\theta v^{1-\Psi}\right) \ominus(\psi(v)+\Phi(v)$ exist $(\forall) \theta>0$, so there exist fuzzy numbers $\omega_{1}(\nu, \theta, \Psi)$, and $\omega_{2}(\nu, \theta, \Psi)$ such that

$$
\psi(v)+\Phi(v)=\left(\psi\left(v+\theta v^{1-\Psi}\right)+\Phi\left(v+\theta v^{1-\Psi}+\omega_{1}(v, \theta, \Psi),\right.\right.
$$


and

$$
\psi\left(v-\theta v^{1-\Psi}\right)+\Phi\left(v-\theta v^{1-\Psi}\right)=\psi(v)+\Phi(v)+\omega_{2}(v, \theta, \Psi) .
$$

Then we have

$$
\psi(v) \ominus \psi\left(v+\theta v^{1-\Psi}\right) \odot(-1) \Phi(v) \odot \Phi\left(\nu+\theta v^{1-\Psi}\right)=\omega_{1}(v, \theta, \Psi) .
$$

Similarly

$$
\psi\left(v-\theta v^{1-\Psi}\right) \ominus \psi(v) \odot \Phi\left(v-\theta v^{1-\Psi}\right) \odot \Phi(v)=\omega_{2}(v, \theta, \Psi) .
$$

Now by multiplying both sides by $\frac{1}{\theta}$, and taking limit both sides, we obtain our required result.

\section{Fuzzy Conformable Derivative of Order $2 \Psi$}

Definition 4.1. A fuzzy-valued function $\Phi$ is strongly generalized differentiable of order $2 \Psi$ if $(\exists)$ a fuzzy number $\Phi^{2 \Psi}(v)$ and

$1(\forall) \theta>0$, there exist H-differences $\Phi^{\Psi}\left(v_{0}+\theta v^{1-\Psi}\right) \ominus \Phi^{\Psi}(v)$ and $\Phi^{\Psi}(v) \ominus \Phi^{\Psi}\left(v-\theta v^{1-\Psi}\right)$ such that

$$
\lim _{\theta \rightarrow 0} \frac{\Phi^{\Psi}\left(v_{0}+\theta v^{1-\Psi}\right) \ominus \Phi^{\Psi}\left(v_{0}\right)}{\theta}=\lim _{\theta \rightarrow 0} \frac{\Phi^{\Psi}\left(v_{0}\right) \ominus \Phi^{\Psi}\left(v_{0} \ominus \theta v^{1-\Psi}\right)}{\theta} .
$$

$2(\forall) \theta>0$, there exist H-differences $\Phi^{\Psi}\left(v_{0}\right) \odot \Phi^{\Psi}\left(v_{0}+\theta v^{1-\Psi}\right)$ and $\Phi^{\Psi}\left(v_{0}-\theta v^{1-\Psi}\right) \Theta$ $\Phi^{\Psi}\left(v_{0}\right)$ such that

$$
\lim _{\theta \rightarrow 0} \frac{\Phi^{\Psi}\left(v_{0}\right) \ominus \Phi^{\Psi}\left(v_{0}+\theta v^{1-\Psi}\right)}{-\theta}=\lim _{\theta \rightarrow 0} \frac{\Phi^{\Psi}\left(v_{0}-\theta v^{1-\Psi}\right) \ominus \Phi^{\Psi}\left(v_{0}\right)}{-\theta} .
$$

1 If $\Phi(v)$, and $\Phi^{\Psi}\left(v_{0}\right)$ both are fuzzy conformable differentiable of the same type, then case (1) of the above definition can be written in the form

$$
\lim _{\theta \rightarrow 0} \frac{\Phi\left(v_{0}+2 \theta v^{1-\Psi}\right)+\Phi\left(v_{0}\right) \odot 2 \Phi\left(v_{0}+\theta v^{1-\Psi}\right)}{\theta^{2}} .
$$

2 If $\Phi\left(v_{0}\right)$, and $\Phi^{\Psi}\left(v_{0}\right)$ are fuzzy conformable differentiable of a different type, then case (2) of the above definition can be written in the form

$$
\lim _{\theta \rightarrow 0} \frac{2 \Phi\left(v_{0}+\theta v^{1-\Psi}\right) \Theta \Phi\left(v_{0}\right)+\Phi\left(v_{0}+2 \theta v^{1-\Psi}\right)}{-\theta^{2}} .
$$

Theorem 4.2. Relation between the strongly generalized conformable differentiable fuzzy-valued function of order $2 \Psi$, and the strongly generalized differentiable fuzzy-valued function of second order is 
1 If $\Phi^{\Psi}$ is the conformable strongly generalized differentiable fuzzy-valued function of the type $(\Psi-1)$, then we have

$$
\Phi_{(1)}^{(2 \Psi)}(v)=v^{2(1-\Psi)} \Phi_{(1)}^{2}(v) .
$$

2 If $\Phi^{\Psi}$ is the conformable strongly generalized differentiable fuzzy-valued function of the type ( $\Psi-2)$, then we have

$$
\Phi_{(2)}^{(2 \Psi)}(v)=v^{2(1-\Psi)} \Phi_{(2)}^{2}(v)
$$

Proof. We prove case (1) here. Case (2) can be proved in a similar pattern easily.

Since we have

$$
\begin{aligned}
& \lim _{\theta \rightarrow 0} \frac{\Phi\left(v_{0}+2 \theta v^{1-\Psi}\right)+\Phi\left(v_{0}\right) \odot 2 \Phi\left(v_{0}+\theta v^{1-\Psi}\right)}{\theta^{2}} \\
= & {\left[\lim _{\theta \rightarrow 0} \frac{\Phi_{*}\left(v_{0}+2 \theta v^{1-\Psi}\right)+\Phi_{*}\left(v_{0}\right)-2 \Phi_{*}\left(v_{0}+\theta v^{1-\Psi}\right)}{\theta^{2}},\right.} \\
& \left.\lim _{\theta \rightarrow 0} \frac{\Phi^{*}\left(v_{0}+2 \theta v^{1-\Psi}\right)+\Phi^{*}\left(v_{0}\right)-2 \Phi^{*}\left(v_{0}+\theta v^{1-\Psi}\right)}{\theta^{2}}\right] .
\end{aligned}
$$

Put $\theta v^{1-\Psi}=\lambda$, we obtain

$$
\begin{aligned}
& \lim _{\theta \rightarrow 0} \frac{\Phi\left(v_{0}+2 \theta v^{1-\Psi}\right)+\Phi\left(v_{0}\right) \odot 2 \Phi\left(v_{0}+\theta v^{1-\Psi}\right)}{\theta^{2}} \\
= & {\left[\lim _{\lambda \rightarrow 0} \frac{\Phi_{*}\left(v_{0}+2 \lambda\right)+\Phi_{*}\left(v_{0}\right)-2 \Phi_{*}\left(v_{0}+\lambda\right)}{\left(\lambda v^{\Psi-1}\right)^{2}},\right.} \\
& \left.\lim _{\lambda \rightarrow 0} \frac{\Phi^{*}\left(v_{0}+2 \lambda\right)+\Phi^{*}\left(v_{0}\right)-2 \Phi^{*}\left(v_{0}+\lambda\right)}{\left(\lambda v^{\Psi-1}\right)^{2}}\right] .
\end{aligned}
$$

We prove the case (1) here. Case (2) can be proved in a similar pattern easily.

Since we have

$$
\begin{aligned}
& \lim _{\theta \rightarrow 0} \frac{\Phi\left(v_{0}+2 \theta v^{1-\Psi}\right)+\Phi\left(v_{0}\right) \odot 2 \Phi\left(v_{0}+\theta v^{1-\Psi}\right)}{\theta^{2}} \\
= & {\left[\lim _{\theta \rightarrow 0} \frac{\Phi_{*}\left(v_{0}+2 \theta v^{1-\Psi}\right)+\Phi_{*}\left(v_{0}\right)-2 \Phi_{*}\left(v_{0}+\theta v^{1-\Psi}\right)}{\theta^{2}},\right.} \\
& \left.\lim _{\theta \rightarrow 0} \frac{\Phi^{*}\left(v_{0}+2 \theta v^{1-\Psi}\right)+\Phi^{*}\left(v_{0}\right)-2 \Phi^{*}\left(v_{0}+\theta v^{1-\Psi}\right)}{\theta^{2}}\right] .
\end{aligned}
$$


Put $\theta v^{1-\Psi}=\lambda$, we obtain

$$
\begin{aligned}
& \lim _{\theta \rightarrow 0} \frac{\Phi\left(v_{0}+2 \theta v^{1-\Psi}\right)+\Phi\left(v_{0}\right) \odot 2 \Phi\left(v_{0}+\theta v^{1-\Psi}\right)}{\theta^{2}} \\
= & {\left[\lim _{\lambda \rightarrow 0} \frac{\Phi_{*}\left(v_{0}+2 \lambda\right)+\Phi_{*}\left(v_{0}\right)-2 \Phi_{*}\left(v_{0}+\lambda\right)}{\left(\lambda v^{\Psi-1}\right)^{2}},\right.} \\
& \left.\lim _{\lambda \rightarrow 0} \frac{\Phi^{*}\left(v_{0}+2 \lambda\right)+\Phi^{*}\left(v_{0}\right)-2 \Phi^{*}\left(v_{0}+\lambda\right)}{\left(\lambda v^{\Psi-1}\right)^{2}}\right] .
\end{aligned}
$$

Then we have

$$
\begin{aligned}
& \lim _{\theta \rightarrow 0} \frac{\Phi\left(v_{0}+2 \theta v^{1-\Psi}\right)+\Phi\left(v_{0}\right) \odot 2 \Phi\left(v_{0}+\theta v^{1-\Psi}\right)}{\theta^{2}} \\
= & v^{2(1-\Psi)}\left[\lim _{\lambda \rightarrow 0} \frac{\Phi_{*}\left(v_{0}+2 \lambda\right)+\Phi_{*}\left(v_{0}\right)-2 \Phi_{*}\left(v_{0}+\lambda\right)}{\lambda^{2}},\right. \\
& \left.\lim _{\lambda \rightarrow 0} \frac{\Phi^{*}\left(v_{0}+2 \lambda\right)+\Phi^{*}\left(v_{0}\right)-2 \Phi^{*}\left(v_{0}+\lambda\right)}{\lambda^{2}}\right] .
\end{aligned}
$$

This gives us our required result in the form

$$
\Phi_{(2)}^{(2 \Psi)}(v)=v^{2(1-\Psi)} \Phi_{(2)}^{2}(v)
$$

Theorem 4.3. Let $\Phi$, and $\psi$ are generalizable conformable $\Psi$-differentiable fuzzy-valued functions, and

1 If $\Phi$ and $\Phi^{\Psi}$ are differentiable of type ( $\left.\Psi-1\right)$, and $\psi, \psi^{\Psi}$ are differentiable of type ( $\Psi$ 2). If H-differences $\Phi(v) \ominus \psi(v)$, and $\Phi^{\Psi}(v) \ominus \psi^{\Psi}(v)$ exists, then $\Phi(v) \ominus \psi(v)$ is strongly generalized differentiable of order $2 \Psi$, and we have

$$
\left(\Phi(v) \ominus \psi(v)^{2 \Psi}=\Phi^{2 \Psi}(v) \ominus \psi^{2 \Psi}(v) .\right.
$$

2 If $\Phi$, and $\psi^{\Psi}$ are differentiable of type ( $\left.\Psi-1\right)$, and $\Phi^{\Psi}, \psi$ are differentiable of type ( $\Psi$ 2). If H-differences $\Phi(v) \ominus \psi(v)$, and $\Phi^{\Psi}(v) \ominus \psi^{\Psi}(v)$ exists, then $\Phi(v) \ominus \psi(v)$ is strongly generalized differentiable of $2 \Psi$, and we have

$$
\left(\Phi(v) \ominus \psi(v)^{2 \Psi}=\Phi^{2 \Psi}(v) \ominus(-1) \psi^{2 \Psi}(v) .\right.
$$

3 If $\Phi$ is differentiable of type ( $\Psi-2)$, and $\Phi^{\Psi}, \psi$, and $\psi^{\Psi}$ are differentiable of type ( $\Psi$ 1). If H-differences $\Phi(v) \odot \psi(v)$, and $\Phi^{\Psi}(v) \odot \psi^{\Psi}(v)$ exists, then $\Phi(v) \ominus \psi(v)$ is strongly generalized differentiable of order $2 \Psi$, and we have

$$
\left(\Phi(v) \ominus \psi(v)^{2 \Psi}=(-1) \Phi^{2 \Psi}(v)+\psi^{2 \Psi}(v) .\right.
$$

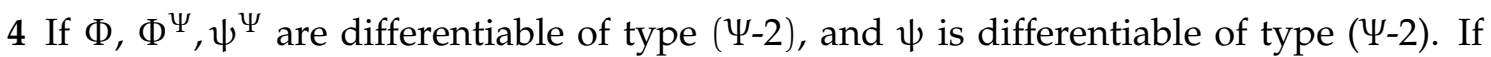
H-differences $\Phi(v) \ominus \psi(v)$, and $\Phi^{\Psi}(v) \ominus \psi^{\Psi}(v)$ exists, then $\Phi(v) \ominus \psi(v)$ is strongly generalized differentiable of order $2 \Psi$, and we have

$$
\left(\Phi(v) \ominus \psi(v)^{2 \Psi}=\Phi^{2 \Psi}(v)+(-1) \psi^{2 \Psi}(v) .\right.
$$


Proof. We provide proof of the case (1) only. Other cases can be proved similarly.

Since $\Phi^{\Psi}$ is differentiable of type $(\Psi-2)$, therefore $\Phi^{\Psi}\left(v+\theta v^{1-\Psi}\right) \ominus \Phi^{\Psi}(v)$ exists, therefore $(\exists)$ a fuzzy number $\eta_{1}^{\Psi}(v, \theta, \Psi)$ such that

$$
\Phi^{\Psi}\left(v+\theta v^{1-\Psi}\right)=\Phi^{\Psi}(v)+\eta_{1}^{\Psi}(v, \theta, \Psi) .
$$

Similarly, Since $\psi^{\Psi}$ is differentiable of type ( $\left.\Psi-2\right)$, there exists $\eta_{2}(\nu, \theta, \Psi)$ such that

$$
\psi^{\Psi}(v)=\psi^{\Psi}\left(v+\theta v^{1-\Psi}\right)+\eta_{2}^{\Psi}(v, \theta, \Psi),
$$

and we have

$$
\begin{aligned}
& \Phi^{\Psi}\left(v+\theta v^{1-\Psi}\right)+(-1) \psi^{\Psi}(v) \\
= & \Phi^{\Psi}(v)+\eta_{1}^{\Psi}(v, \theta, \Psi)+(-1) \psi^{\Psi}\left(v+\theta v^{1-\Psi}\right)+(-1) \eta_{2}^{\Psi}(v, \theta, \Psi) .
\end{aligned}
$$

Since the H-difference $\Phi^{\Psi}\left(v+\theta v^{1-\Psi}\right)+(-1) \psi^{\Psi}\left(v+\theta v^{1-\Psi}\right) \ominus \Phi^{\Psi}(v)+(-1) \psi^{\Psi}(v)$ exists, so we have

$$
\begin{aligned}
& \Phi^{\Psi}\left(v+\theta v^{1-\Psi}\right)+(-1) \psi^{\Psi}\left(v+\theta v^{1-\Psi}\right) \ominus \Phi^{\Psi}(v)+(-1) \psi^{\Psi}(v) \\
= & \eta_{1}^{\Psi}(v, \theta, \Psi) \ominus(-1) \eta_{2}^{\Psi}(v, \theta, \Psi) .
\end{aligned}
$$

Similarly, $(\exists) v_{1}^{\Psi}(v, \theta, \Psi)$, and $v_{2}^{\Psi}(v, \theta, \Psi)$, such that

$$
\begin{aligned}
\Phi^{\Psi}(v) & =\Phi^{\Psi}\left(v-\theta v^{1-\Psi}\right)+v_{1}^{\Psi}(v, \theta, \Psi), \\
\psi^{\Psi}\left(v-\theta v^{1-\Psi}\right) & =\psi^{\Psi}(v)+v_{2}^{\Psi}(v, \theta, \Psi),
\end{aligned}
$$

and therefore we get

$$
\begin{aligned}
& \left(\Phi^{\Psi}(v)+(-1) \Psi^{\Psi}(v) \ominus \Phi^{\Psi}\left(v-\theta v^{1-\Psi}\right)+(-1) \Psi^{\Psi}\left(v-\theta v^{1-\Psi}\right)\right. \\
= & \eta_{2}^{\Psi}(v, \theta, \Psi) \ominus(-1) v_{2}^{\Psi}(v, \theta, \Psi) .
\end{aligned}
$$

Note that

$$
\lim _{\theta \rightarrow 0} \frac{\eta_{1}^{\Psi}(v, \theta, \Psi)}{\theta}=\lim _{\theta \rightarrow 0} \frac{\eta_{2}^{\Psi}(v, \theta, \Psi)}{\theta}=\Phi^{2 \Psi}(v)
$$

Also

$$
\lim _{\theta \rightarrow 0} \frac{v_{1}^{\Psi}(v, \theta, \Psi)}{\theta}=\lim _{\theta \rightarrow 0} \frac{v_{2}^{\Psi}(v, \theta, \Psi)}{\theta}=(-1) \psi^{2 \Psi}(v) .
$$

Thus we obtain our required result.

$$
(\Phi(v) \ominus \psi(v))^{2 \Psi}=\Phi^{2 \Psi}(v)+(-1) \psi^{2 \Psi}(v) .
$$

Example 4.4. Consider strongly generalized differentiable fuzzy-valued functions $\Phi$, and $\psi$ of order $2 \Psi$ defined as $\Phi(v)=(1,5,7) \odot\left(\frac{v^{\Psi}}{\Psi}\right)^{2}, \psi(v)=(2,4,6) \odot \sin \left(\frac{v^{\Psi}}{\Psi}\right)$.

1. Since $\Phi(v), \Phi^{\Psi}(v)$ are differentiable of type $(\Psi-1)$ and $\psi(v), \psi^{\Psi}(v)$ is differentiable of type ( $\Psi-2)$. So applying case (1) of the above theorem, we obtain

$$
\begin{aligned}
& (\Phi \odot \psi)^{2 \Psi}\left(\frac{\nu^{\Psi}}{\Psi}\right) \\
= & {[2+8 \gamma, 14-4 \gamma] \ominus\left(-\sin \frac{\nu^{\Psi}}{\Psi}\right) \cdot[2+2 \gamma, 6-2 \gamma],(\forall) v \in(0, \infty] . }
\end{aligned}
$$


2. Note that here $\Phi(v), \psi^{\Psi}(v)$ are differentiable of type $(\Psi-1)$ and $\Phi^{\Psi}(v)$, $\psi(v)$ is differentiable of type ( $\Psi-2)$, so applying case (2) of the above theorem, we obtain

$$
\begin{aligned}
& (\Phi \ominus \psi)^{2 \Psi}\left(\frac{\nu^{\Psi}}{\Psi}\right) \\
= & \left(-\sin \frac{\nu^{\Psi}}{\Psi}\right) \cdot[2+2 \gamma, 6-2 \gamma] \ominus[2+8 \gamma, 14-4 \gamma],(\forall) v \in(0, \infty] .
\end{aligned}
$$

3. If we have the situation where $\Phi(v)$ is differentiable of type $(\Psi-1)$ and $\Phi^{\Psi}(v), \psi(v)$, $\psi^{\Psi}(v)$ are differentiable of type ( $\left.\Psi-2\right)$, then applying case (3) of the above theorem, we obtain

$$
\begin{aligned}
& (\Phi \ominus \psi)^{2 \Psi}\left(\frac{\nu^{\Psi}}{\Psi}\right) \\
= & (-1)[2+8 \gamma, 14-4 \gamma]+\left(-\sin \frac{\nu^{\Psi}}{\Psi}\right) \cdot[2+2 \gamma, 6-2 \gamma],(\forall) v \in(0, \infty] .
\end{aligned}
$$

4. While if $\Phi(v), \Phi^{\Psi}(v), \psi(v),\left(\frac{v^{\Psi}}{\Psi}\right)^{2}$ are differentiable of type $(\Psi-1)$ and $\psi^{\Psi}(v)$ is differentiable of type ( $\Psi-2)$, then applying case (4) of the above theorem, we obtain

$$
(\Phi \ominus \psi)^{2 \Psi}\left(\frac{\nu^{\Psi}}{\Psi}\right)=-[2+2 \gamma, 6-2 \gamma] \cdot\left(-\sin \frac{\nu^{\Psi}}{\Psi}\right)+(-1)[2+8 \gamma, 14-4 \beta],(\forall) v \in(0, \infty] .
$$

Definition 4.5. A fuzzy-valued function $\Phi$ is differentiable of type

1. ( $\Psi-1)$ if $\Phi$ is differentiable in the case (1) of definition (4.1).

2. ( $\Psi-2)$ if $\Phi$ is differentiable in the case (2) of definition (4.1).

Theorem 4.6. ([29]) Let $\Phi$ is a fuzzy-valued function with $\gamma$-cuts representation $[\Phi(v)]^{\gamma}=$ $\left[\left(\Phi_{*}\right)_{\gamma}(v),\left(\Phi^{*}\right)_{\gamma}(v)\right]$. When we take a strongly generalized conformable derivative of a fuzzyvalued function of order $\Psi$, we have two possible cases. $\Phi_{1}^{\Psi-1}(v)$, and $\Phi_{2}^{\Psi-1}(v)$. For the derivative of order $2 \Psi$, we have again two possible cases on each $\Psi$-derivative, which are

1 If $\Phi_{1}^{\Psi}$ is differentiable of type $(\Psi-1)$, then $\left(\Phi_{*}\right)_{\gamma}$, and $\left(\Phi^{*}\right)_{\gamma}$ are $\Psi$-differentiable, and we have

$$
\left[\Phi^{2 \Psi}(v)\right]^{\gamma}=\left[\left(\Phi_{*}^{2 \Psi}\right)_{\gamma}(v),\left(\Phi^{* 2 \Psi}\right)_{\gamma}(v)\right] .
$$

2. If $\Phi_{1}^{\Psi}$ is differentiable of type $(\Psi-2)$, then $\left(\Phi^{*}\right)_{\gamma}$, and $\left(\Phi_{*}\right)_{\gamma}$ are $\Psi$-differentiable, and we have

$$
\left[\Phi^{2 \Psi}(v)\right]^{\gamma}=\left[\left(\Phi^{* 2 \Psi}\right)_{\gamma}(v),\left(\Phi_{*}^{2 \Psi}\right)_{\gamma}(v)\right] .
$$

3. If $\Phi_{2}^{\Psi}$ is differentiable of type $(\Psi-1)$, then $\left(\Phi^{*}\right)_{\gamma}$, and $\left(\Phi_{*}\right)_{\gamma}$ are $\Psi$-differentiable, and we have

$$
\left[\Phi^{2 \Psi}(v)\right]^{\gamma}=\left[\left(\Phi^{* 2 \Psi}\right)_{\gamma}(v),\left(\Phi_{*}^{2 \Psi}\right)_{\gamma}(v)\right]
$$


4. If $\Phi_{2}^{\Psi}$ is differentiable of type $(\Psi-2)$, then $\left(\Phi_{*}\right)_{\gamma^{\prime}}$ and $\left(\Phi^{*}\right)_{\gamma}$ are $\Psi$-differentiable, and we have

$$
\left[\Phi^{2 \Psi}(\nu)\right]^{\gamma}=\left[\left(\Phi_{*}^{2 \Psi}\right)_{\gamma}(\nu),\left(\Phi^{* 2 \Psi}\right)_{\gamma}(v)\right] .
$$

Theorem 4.7. Suppose $\Phi$, and $\psi$ are fuzzy-valued functions which are strongly generalized differentiable of order $p \Psi$ at $v$ in the same case, then $\Phi+\psi$ is differentiable of order $p \Psi a t v$, and

$$
(\Phi+\psi)^{p \Psi}(v)=\Phi^{p \Psi}(v)+\psi^{p \Psi}(v) .
$$

Theorem 4.8. If $\Phi$, and $\psi$ are conformable strongly generalized differentiable fuzzy-valued functions of order $2 \Psi$ such that $\Phi$ is differentiable in the form $(\Psi-1)$, and $\Psi$ is differentiable in the form $(\Psi-3)$ or $\Phi$ is strongly generalized differentiable in the form $(\Psi-2)$, and $\Psi$ is differentiable in the form ( $\Psi-4)$ or $\Phi$ is differentiable in the form ( $\Psi-3)$, and $\Psi$ is differentiable in the form $(\Psi-1)$ or $\Phi$ is differentiable in the form $(\Psi-4)$, and $\psi$ is differentiable in the form $(\Psi-2)$. If $\mathrm{H}$-difference $\Phi(v) \ominus \psi(v)$ exists for $v \in(0, \infty), \Phi \ominus \psi$ is strongly generalized differentiable of order $2 \Psi$, and

$$
(\Phi \ominus \psi)^{2 \Psi}(v)=\Phi^{2 \Psi}(v)+(-1) \psi^{2 \Psi}(v) .
$$

Proof. We prove the case (1). Other cases can be proved in a similar pattern.

If $\Phi$ is differentiable of type ( $\Psi-1)$, and $\psi$ is differentiable of type ( $\Psi-2)$, then we have $\Phi \ominus \psi(\nu)$ is differentiable of type ( $\Psi-1)$, and

$$
(\Phi \ominus \psi)^{\Psi}(v)=\Phi^{\Psi}(v)+(-1) \psi^{\Psi}(v) .
$$

Now if we differentiate $\Phi$ in the form ( $\Psi-1)$, and differentiate $\psi$ in the form $(\Psi-2)$, then by theorem, $\Phi \ominus \psi(v)$ is differential of type ( $\Psi-1)$ differentiable, and we have our required result,

$$
(\Phi \odot \psi)^{2 \Psi}(v)=\left(\Phi^{\Psi}(v)+(-1) \psi^{\Psi}(v)\right)^{\Psi}=\Phi^{2 \Psi}(v)+(-1) \psi^{2 \Psi}(v) .
$$

Theorem 4.9. Let $\Phi:(0, \infty) \rightarrow \mathbb{R}$, and $\psi:(0, \infty) \rightarrow \mathbb{R}_{\Phi}$ are differentiable functions of order $2 \Psi$.

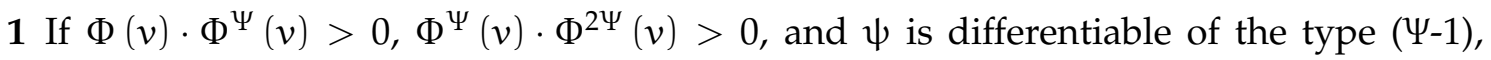
then $\Phi$ and $\psi$ are $\Psi$-differentiable of the type ( $\Psi-1)$, and we have

$$
(\Phi \odot \psi)^{2 \Psi}(v)=\Phi^{2 \Psi}(v) \odot \psi(v)+2 \Phi^{\Psi}(v) \odot \psi^{\Psi}(v)+\Phi(v) \odot \psi^{2 \Psi}(v) .
$$

2 If $\Phi(v) \cdot \Phi^{\Psi}(v)<0, \Phi^{\Psi}(v) \cdot \Phi^{2 \Psi}(v)<0$, and $\psi$ is differentiable of the type ( $\left.\Psi-4\right)$, then $\Phi .$, and $\psi$ are $\Psi$-differentiable of the type ( $\Psi-2)$, and we have

$$
(\Phi \odot \psi)^{2 \Psi}(v)=\Phi^{2 \Psi}(v) \odot \psi(v)+2 \Phi^{\Psi}(v) \odot \psi^{\Psi}(v)+\Phi(v) \odot \psi^{2 \Psi}(v) .
$$

Proof. If $\Phi(v) \cdot \Phi^{\Psi}(v)>0$, and $\psi$ is differentiable of type ( $\left.\Psi-1\right)$, then we have

$$
(\Phi \odot \psi)^{\Psi}(v)=\Phi^{\Psi}(v) \odot \psi(v)+\Phi(v) \odot \psi^{\Psi}(v) .
$$

Now if we differentiate $\psi(v)$ in the form $(\Psi-1)$, and $\Phi(v) \cdot \Phi^{\Psi}(v)>0$, then we obtain our result. 
Theorem 4.10. Let $\psi:(0, \infty) \rightarrow \mathbb{R}$ be a conformable differentiable function of order $2 \Psi$, and $\eta$ $\in \mathbb{R}_{\Phi}$. We define $\Phi:(0, \infty) \rightarrow \mathbb{R}_{\Phi}$ by $\Phi(v)=\eta \odot \psi(v)$.

1 If $\psi(v) \cdot \psi^{\Psi}(v)>0$, and $\psi^{\Psi}(v) \cdot \psi^{2 \Psi}(v)>0$, then $\Phi(v)$ is differentiable of the type $(\Psi-1)$, and it's derivative of order $2 \Psi$ is

$$
\Phi_{(1)}^{2 \Psi}(v)=\eta \odot \psi^{2 \Psi}(v) .
$$

2 If $\psi(v) \cdot \psi^{\Psi}(v)>0$, and $\psi^{\Psi}(v) \cdot \psi^{2 \Psi}(v)<0$, then $\Phi(v)$ is differentiable of the type $(\Psi-2)$, and it's derivative of order $2 \Psi$ is

$$
\Phi_{(2)}^{2 \Psi}(v)=\eta \odot \psi^{2 \Psi}(v) .
$$

3. If $\psi(v) \cdot \psi^{\Psi}(v)<0$, and $\psi^{\Psi}(v) \cdot \psi^{2 \Psi}(v)>0$, then $\Phi(v)$ is differentiable of the type $(\Psi-3)$, and it's derivative of order $2 \Psi$ is

$$
\Phi_{(3)}^{2 \Psi}(v)=\eta \odot \psi^{2 \Psi}(v) .
$$

4. If $\psi(v) \cdot \psi^{\Psi}(v)<0$, and $\psi^{\Psi}(v) \cdot \psi^{2 \Psi}(v)<0$, then $\Phi(v)$ is differentiable of the type $(\Psi-4)$, and it's derivative of order $2 \Psi$ is

$$
\Phi_{(4)}^{2 \Psi}(v)=\eta \odot \psi^{2 \Psi}(v) .
$$

Proof. We prove case (1) only. Other cases can be easily proved with the same technique. By Theorem 3.18, if we define

$$
\Phi(v)=\eta \odot \psi(v),
$$

and $\psi(v) \cdot \psi^{\Psi}(v)>0$, then $\Phi$ is differentiable of type ( $\left.\Psi-1\right)$, and we have

$$
\Phi^{\Psi}(v)=\eta \odot \psi^{\Psi}(v) .
$$

Now since we have $\psi^{\Psi}(v) \cdot \psi^{2 \Psi}(v)>0$, then applying Theorem 3.18, we have $\Phi$ is differentiable of the type $(\Psi-1)$, and it's derivative of order $2 \Psi$ is

$$
\Phi_{(1)}^{2 \Psi}(v)=\eta \odot \psi^{2 \Psi}(v) .
$$

Example 4.11. Define a fuzzy-valued function in the form

$$
\Phi(v)=\eta \odot \psi(v)
$$

where $\eta$ is a fuzzy number, and $\psi$ is a $\left(2, \frac{1}{2}\right)$ polynomial given by

$$
\psi(v)=v^{2}-3 v^{\frac{3}{2}}+5 v-6,
$$

and $v$ ranges from 0 to 4 . Now, we have five possibilities. 
1 We have $\psi(v) \cdot \psi^{\Psi}(v)<0$, and $\psi^{\Psi}(v) \cdot \psi^{2 \Psi}(v)<0$ for $v \in(0,1)$, so by applying the above theorem, $\Phi(v)$ is differentiable of type $(\Psi-4)$, and it's derivative is

$$
\Phi^{2 \Psi}(v)=2 v^{\frac{5}{2}}-\frac{9}{2} v+\frac{5}{2} v^{\frac{1}{2}} \odot \eta
$$

2. We have $\psi(v) \cdot \psi^{\Psi}(v)>0$, and $\psi^{\Psi}(v) \cdot \psi^{2 \Psi}(v)<0$ for $v \in(1,2)$, so by applying the above theorem, $\Phi(v)$ is differentiable of type $(\Psi-2)$, and it's derivative is

$$
\Phi^{2 \Psi}(v)=2 v^{\frac{5}{2}}-\frac{9}{2} v+\frac{5}{2} v^{\frac{1}{2}} \odot \eta .
$$

3. We have $\psi(v) \cdot \psi^{\Psi}(v)<0$, and $\psi^{\Psi}(v) \cdot \psi^{2 \Psi}(v)>0$ for $v \in(2,3)$, so by applying the above theorem, $\Phi(v)$ is differentiable of type ( $\Psi-3)$, and it's derivative is

$$
\Phi^{2 \Psi}(v)=2 v^{\frac{5}{2}}-\frac{9}{2} v+\frac{5}{2} v^{\frac{1}{2}} \odot \eta
$$

4. We have $\psi(v) \cdot \psi^{\psi}(v)>0$, and $\psi^{\psi}(v) \cdot \psi^{2 \Psi}(v)>0$ for $v \in(3,4)$, so applying the above theorem, $\Phi(\nu)$ is differentiable of type ( $\Psi-1)$, and it's derivative is

$$
\Phi^{2 \Psi}(v)=2 v^{\frac{5}{2}}-\frac{9}{2} v+\frac{5}{2} v^{\frac{1}{2}} \odot \eta
$$

5. We have $\psi^{\Psi}(v) \cdot \psi^{2 \Psi}(v)=0$ for $v=1$, so by applying the above theorem, $\Phi(v)$ is differentiable of order $2 \Psi$, and it's derivative is

$$
\Phi^{2 \Psi}(v)=2 v^{\frac{5}{2}}-\frac{9}{2} v+\frac{5}{2} v^{\frac{1}{2}} \odot \eta
$$

\section{Fuzzy Conformable Derivative of Order P $\Psi$}

Throughout this subsection, we will use the terms given in the form ([28])

$$
\begin{aligned}
& \digamma(\Phi(v)=\Phi(v) \text { if } \Phi(v) \text { is differentiable of type ( } \Psi-1) \text {, }
\end{aligned}
$$

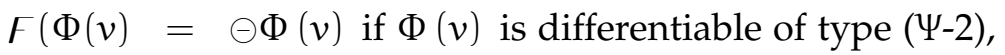

and

$$
\begin{aligned}
& \digamma^{p^{\Psi}}\left(\Phi(v)=\Phi^{(p-1)(\Psi)}(v) \text { if } \Phi^{(p-1)(\Psi)}(v) \text { is differentiable of type }(\Psi-1),\right. \\
& \digamma^{p \Psi}\left(\Phi(v)=\Theta \Phi^{(p-1)(\Psi)}(v) \text { if } \Phi^{(p-1)(\Psi)}(v) \text { is differentiable of type }(\Psi-2),\right.
\end{aligned}
$$

where $\digamma^{p(\Psi)}(v)=\digamma\left(\digamma^{(p-1)(\Psi)}(v)\right.$.

Definition 5.1. A fuzzy-valued function $\Phi$ is conformable strongly generalized differentiable of order $p \Psi$ at $v_{0} \in^{\prime}(a, b)$ if $(\exists) \Phi^{p^{\Psi}}\left(v_{0}\right) \in \mathbb{R}_{\Phi}$ such that

$1(\forall) \theta>0$, there exist H-differences $\Phi^{(p-1) \Psi}\left(v_{0}+\theta v^{1-\Psi}\right) \ominus \Phi^{(p-1) \Psi}\left(v_{0}\right), \Phi^{(p-1) \Psi}\left(v_{0}\right) \ominus$ $\Phi^{(p-1) \Psi}\left(v_{0} \ominus \theta v^{1-\Psi}\right)$ such that

$$
\lim _{\theta \rightarrow 0} \frac{\Phi^{(p-1) \Psi}\left(v_{0}+\theta v^{1-\Psi}\right) \ominus \Phi^{(p-1) \Psi}\left(v_{0}\right)}{\theta^{p}}=\lim _{\theta \rightarrow 0} \frac{\Phi^{(p-1) \Psi}\left(v_{0}\right) \ominus \Phi^{(p-1) \Psi}\left(v_{0} \ominus \theta v^{1-\Psi}\right)}{\theta^{p}} .
$$


$2(\forall) \theta>0$, there exist H-differences $\Phi^{(p-1) \Psi}\left(v_{0}\right) \ominus \Phi^{(p-1) \Psi}\left(v_{0}+\theta v^{1-\Psi}\right), \Phi^{(p-1) \Psi}\left(v_{0}-\right.$ $\left.\theta v^{1-\Psi}\right) \ominus \Phi^{(p-1) \Psi}\left(v_{0}\right)$

$$
\lim _{\theta \rightarrow 0} \frac{\Phi^{(p-1) \Psi}\left(v_{0}\right) \ominus \Phi^{(p-1) \Psi}\left(v_{0}+\theta v^{1-\Psi}\right)}{-\theta^{p}}=\lim _{\theta \rightarrow 0} \frac{\Phi^{(p-1) \Psi}\left(v_{0}-\theta v^{1-\Psi}\right) \ominus \Phi^{(p-1) \Psi}\left(v_{0}\right)}{-\theta^{p}} .
$$

Remark 5.2. For $p=1$, the above definition becomes equal to the definition of the fuzzy conformable strongly generalized derivative of order $\Psi$.

Theorem 5.3. Above definition has four cases associated with odd and even values of $\mathrm{p}$.

Case 1: If $\mathrm{p}$ is even, then we have two possibilities.

If $\Phi^{i \Psi}\left(v_{0}\right)$ is differentiable of type ( $\left.\Psi-1\right)$ for even values of $i$ for $i=1,2, \cdots p$, and differentiable of type ( $\Psi-2)$ for other values in the above definition, then

$$
\begin{aligned}
& \Phi^{p^{\Psi}}\left(v_{0}\right)=\lim _{\theta \rightarrow 0} \frac{\sum_{i=0}^{p}\left(\begin{array}{c}
p \\
i
\end{array}\right)\left(\digamma ^ { i \Psi } \left(\Phi\left(v_{0}+(p-i) \theta v^{1-\Psi}\right)\right.\right.}{\theta^{p}}, \\
& \Phi^{p^{\Psi}}\left(v_{0}\right)=\lim _{\theta \rightarrow 0} \frac{\sum_{i=0}^{p}\left(\begin{array}{c}
p \\
i
\end{array}\right)\left(\digamma ^ { i \Psi } \left(\Phi\left(v_{0}+(p-i-1) \theta v^{1-\Psi}\right)\right.\right.}{\theta^{p}}, \\
& \Phi^{p \Psi}\left(v_{0}\right)=\lim _{\theta \rightarrow 0} \frac{\sum_{i=0}^{p}\left(\begin{array}{c}
p \\
i
\end{array}\right)\left(\digamma ^ { i \Psi } \left(\Phi\left(v_{0}+(p-i-2) \theta v^{1-\Psi}\right)\right.\right.}{\theta^{p}} .
\end{aligned}
$$

Continuing in this way,finally we reach the step

$$
\Phi^{p \Psi}\left(v_{0}\right)=\lim _{\theta \rightarrow 0} \frac{\sum_{i=0}^{p}\left(\begin{array}{c}
p \\
i
\end{array}\right)\left(\digamma ^ { i \Psi } \left(\Phi\left(v_{0}+\left(p-i \theta v^{1-\Psi}\right)\right)\right.\right.}{\theta^{p}} .
$$

If $\Phi^{i \Psi}\left(v_{0}\right)$ is differentiable of type ( $\left.\Psi-1\right)$ for odd values of $i$ for $i=1,2, \cdots p-1$, and differentiable of type ( $\Psi-2)$ for other values in above definition, then

$$
\begin{aligned}
& \Phi^{\mathrm{p} \Psi}\left(v_{0}\right)=\lim _{\theta \rightarrow 0} \frac{\sum_{i=0}^{p}\left(\begin{array}{c}
p \\
i
\end{array}\right)\left(\digamma^{i \Psi}\left(\Phi\left(v_{0}+i \theta v^{1-\Psi}\right)\right)\right.}{\sum^{p}\left(\begin{array}{c}
p \\
i
\end{array}\right)\left(\digamma^{i \Psi}\left(\Phi\left(v_{0}+(i-1) \theta v^{1-\Psi}\right)\right)\right.} \\
& \Phi^{p \Psi}\left(v_{0}\right)=\lim _{\theta \rightarrow 0} \frac{\sum_{i=0}^{p}\left(\begin{array}{c}
p \\
i
\end{array}\right)\left(\digamma^{i \Psi}\left(\Phi\left(v_{0}+(i-2) \theta v^{1-\Psi}\right)\right)\right.}{-\theta^{p}} \\
& \Phi^{p \Psi}\left(v_{0}\right)=\lim _{\theta \rightarrow 0} \frac{{ }_{i=0}^{p}}{}
\end{aligned}
$$

The above definition has two cases associated with odd, and even values of $p$.

Theorem 5.4. Case 1: If $\mathrm{p}$ is even, then we have four possibilities. 
(i) If $\Phi^{i \Psi}\left(v_{0}\right)$ is differentiable of type ( $\left.\Psi-1\right)$ for even values of $i$ for $i=1,2, \cdots p$, and differentiable of type ( $\Psi-2)$ for other values in the above definition, then

$$
\begin{aligned}
& \Phi^{p^{\Psi}}\left(v_{0}\right)=\lim _{\theta \rightarrow 0} \frac{\sum_{i=0}^{p}\left(\begin{array}{c}
p \\
i
\end{array}\right)\left(\digamma ^ { i \Psi } \left(\Phi\left(v_{0}+(p-i) \theta v^{1-\Psi}\right)\right.\right.}{\theta^{p}}, \\
& \Phi^{p^{\Psi}}\left(v_{0}\right)=\lim _{\theta \rightarrow 0} \frac{\sum_{i=0}^{p}\left(\begin{array}{c}
p \\
i
\end{array}\right)\left(\digamma ^ { i \Psi } \left(\Phi\left(v_{0}+(p-i-1) \theta v^{1-\Psi}\right)\right.\right.}{\theta^{p}}, \\
& \Phi^{p^{\Psi}}\left(v_{0}\right)=\lim _{\theta \rightarrow 0} \frac{\sum_{i=0}^{p}\left(\begin{array}{c}
p \\
i
\end{array}\right)\left(\digamma ^ { i \Psi } \left(\Phi\left(v_{0}+(p-i-2) \theta v^{1-\Psi}\right)\right.\right.}{\theta^{p}},
\end{aligned}
$$

continuing in this way, finally, we reach the step

$$
\Phi^{p \Psi}\left(v_{0}\right)=\lim _{\theta \rightarrow 0} \frac{\sum_{i=0}^{p}\left(\begin{array}{c}
p \\
i
\end{array}\right)\left(\digamma ^ { i \Psi } \left(\Phi\left(v_{0}+\left(p-i \theta v^{1-\Psi}\right)\right)\right.\right.}{\theta^{p}} .
$$

(ii) If $\Phi^{i \Psi}\left(v_{0}\right)$ is differentiable of type ( $\left.\Psi-1\right)$ for odd values of $i$ for $i=1,2, \cdots p-1$, and differentiable of type ( $\Psi-2)$ for other values in above definition, then

$$
\begin{aligned}
& \Phi^{\mathrm{p} \Psi}\left(v_{0}\right)=\lim _{\theta \rightarrow 0} \frac{\sum_{i=0}^{p}\left(\begin{array}{c}
p \\
i
\end{array}\right)\left(\digamma^{i \Psi}\left(\Phi\left(v_{0}+i \theta v^{1-\Psi}\right)\right)\right.}{-\theta^{p}}, \\
& \Phi^{p \Psi}\left(v_{0}\right)=\lim _{\theta \rightarrow 0} \frac{\sum_{i=0}^{p}\left(\begin{array}{c}
p \\
i
\end{array}\right)\left(\digamma^{i \Psi}\left(\Phi\left(v_{0}+(i-1) \theta v^{1-\Psi}\right)\right)\right.}{-\theta^{p}}, \\
& \Phi^{p \Psi}\left(v_{0}\right)=\lim _{\theta \rightarrow 0} \frac{\sum_{i=0}^{p}\left(\begin{array}{c}
p \\
i
\end{array}\right)\left(\digamma^{i \Psi}\left(\Phi\left(v_{0}+(i-2) \theta v^{1-\Psi}\right)\right)\right.}{-\theta^{p}}
\end{aligned}
$$

continuing in this way, finally, we reach the step

$$
\Phi^{p \Psi}\left(v_{0}\right)=\lim _{\theta \rightarrow 0} \frac{\sum_{i=0}^{p}\left(\begin{array}{c}
p \\
i
\end{array}\right)\left(\digamma ^ { i \Psi } \left(\Phi\left(v_{0}+(i-p+1) \theta v^{1-\Psi}\right)\right.\right.}{-\theta^{p}} .
$$

Case 2: For odd values of $\mathrm{p}$, we have two possibilities.

(i) If $\Phi^{i \Psi}\left(v_{0}\right)$ is differentiable of type ( $\left.\Psi-1\right)$ for odd values of $i$ for $i=1,2, \cdots p$, and differentiable of type $(\Psi-2)$ for other values in the above definition, then

$$
\begin{aligned}
& \Phi^{p^{\Psi}}\left(v_{0}\right)=\lim _{\theta \rightarrow 0} \frac{\sum_{i=0}^{p}\left(\begin{array}{c}
p \\
i
\end{array}\right)\left(\digamma ^ { i \Psi } \left(\Phi\left(v_{0}+(p-i) \theta v^{1-\psi}\right)\right.\right.}{\theta^{p}}, \\
& \Phi^{p \Psi}\left(v_{0}\right)=\lim _{\theta \rightarrow 0} \frac{\sum_{i=0}^{p}\left(\begin{array}{c}
p \\
i
\end{array}\right)\left(\digamma ^ { i \Psi } \left(\Phi\left(v_{0}+(p-i-1) \theta v^{1-\psi}\right)\right.\right.}{\theta^{p}}, \\
& \Phi^{p^{\Psi}}\left(v_{0}\right)=\lim _{\theta \rightarrow 0} \frac{\sum_{i=0}^{p}\left(\begin{array}{c}
p \\
i
\end{array}\right)\left(\digamma ^ { i \Psi } \left(\Phi\left(v_{0}+(p-i-2) \theta v^{1-\psi}\right)\right.\right.}{\theta^{p}},
\end{aligned}
$$


continuing in this way, finally, we reach the step

$$
\Phi^{\mathrm{p} \Psi}\left(v_{0}\right)=\lim _{\theta \rightarrow 0} \frac{\sum_{i=0}^{p}\left(\begin{array}{c}
p \\
i
\end{array}\right)\left(\digamma ^ { i \Psi } \left(\Phi\left(v_{0}-i \theta v^{1-\Psi}\right)\right.\right.}{\theta^{p}} .
$$

(ii) If $\Phi^{i \Psi}\left(v_{0}\right)$ is differentiable of type ( $\left.\Psi-1\right)$ for even values of $i$ for $i=1,2, \cdots p-1$, and differentiable of type ( $\Psi-2)$ for other values in the above definition, then

$$
\begin{aligned}
& \Phi^{p^{\Psi}}\left(v_{0}\right)=\lim _{\theta \rightarrow 0} \frac{\sum_{i=0}^{p}\left(\begin{array}{c}
p \\
i
\end{array}\right)\left(\digamma ^ { i \Psi } \left(\Phi\left(v_{0}+i \theta v^{1-\Psi}\right)\right.\right.}{-\theta^{p}}, \\
& \Phi^{p^{\Psi}}\left(v_{0}\right)=\lim _{\theta \rightarrow 0} \frac{\sum_{i=0}^{p}\left(\begin{array}{c}
p \\
i
\end{array}\right)\left(\digamma ^ { i \Psi } \left(\Phi\left(v_{0}+(i-1) \theta v^{1-\Psi}\right)\right.\right.}{-\theta^{p}}, \\
& \Phi^{p \Psi}\left(v_{0}\right)=\lim _{\theta \rightarrow 0} \frac{\sum_{i=0}^{p}\left(\begin{array}{c}
p \\
i
\end{array}\right)\left(\digamma ^ { i \Psi } \left(\Phi\left(v_{0}+(i-2) \theta v^{1-\Psi}\right)\right.\right.}{-\theta^{p}}
\end{aligned}
$$

continuing in this way, finally, we reach the step

$$
\Phi^{p \Psi}\left(v_{0}\right)=\lim _{\theta \rightarrow 0} \frac{\sum_{i=0}^{p}\left(\begin{array}{l}
p \\
i
\end{array}\right)\left(\digamma ^ { i \Psi } \left(\Phi\left(v_{0}+(i-p+1) \theta v^{1-\Psi}\right)\right.\right.}{-\theta^{p}} .
$$

Remark 5.5. If we put $p=1$ in the above equation, then we get the definition of the strongly generalized conformable derivative of order $\Psi$.

Theorem 5.6. Define a fuzzy-valued function by

$$
\Phi(v)=\eta \odot \psi(v),
$$

where $\eta$ is a fuzzy number. Now if $\psi$ is a differentiable real-valued function of order $p \Psi$, then $\Phi$ is the strongly generalized differentiable of order $\mathrm{p} \Psi$, and we have

$$
\Phi^{p^{\Psi}}(v)=\eta \odot \psi^{p \Psi}(v) .
$$

Theorem 5.7. If $\mathrm{p}$ is odd, then we have four cases.

1. If a kth element of $\Phi^{i \Psi}(v)$ is differentiable of type ( $\left.\Psi-1\right)$ for odd values of $i$, and differentiable of type ( $\Psi-2)$ for other values, and an ith element of $\psi^{j \Psi}(\nu)$ is differentiable of type ( $\Psi-1)$ for odd values of $j$. Then $\Phi(v)+\psi(v)$ is differentiable of order $\mathrm{p} \Psi$, and we have

$$
(\Phi(v)+\psi(v))^{p \Psi}=\Phi^{p \Psi}(v) \odot(-1) \psi^{p \Psi}(v) .
$$

2. If a kth element of $\Phi^{i \Psi}(v)$ is differentiable of type ( $\left.\Psi-1\right)$ for even values of $i$, and an ith element of $\psi^{j \Psi}(v)$ is differentiable of type ( $\left.\Psi-1\right)$ for odd values of $j$. Then $\Phi(v)+\psi(v)$ is differentiable of order $p \Psi$, and we have

$$
(\Phi(v)+\psi(v))^{p \Psi}=(-1) \psi^{p \Psi}(v)+(-1) \Phi^{p \Psi}(v) .
$$


3. If a kth element of $\Phi^{i \Psi}(v)$ is differentiable of type ( $\left.\Psi-1\right)$ for even values of $i$, and differentiable of type $(\Psi-2)$ for other values, and an ith element of $\psi^{j \Psi}(v)$ is differentiable of type $(\Psi-1)$ for even values of $j$. Then $\Phi(v)+\psi(v)$ is differentiable of order $\mathrm{p} \Psi$, and we have

$$
(\Phi(v)+\psi(v))^{p \Psi}=\psi^{p \Psi}(v) \ominus(-1) \Phi^{p \Psi}(v) .
$$

4. If a kth element of $\Phi^{i \Psi}(v)$ is differentiable of type ( $\left.\Psi-1\right)$ for odd values of $i$, and differentiable of type ( $\Psi-2)$ for other values, and an ith element of $\psi^{j \Psi}(\nu)$ is differentiable of type ( $\Psi-1)$ for odd values of $j$. Then $\Phi(v)+\psi(v)$ is differentiable of order $\mathrm{p} \Psi$, and we have

$$
(\Phi(v)+\psi(v))^{\mathrm{p} \Psi}=\Phi^{\mathrm{p} \Psi}(v)+\psi^{\mathrm{p} \Psi}(v) .
$$

1. If a kth element of $\Phi^{i \Psi}(v)$ is differentiable of type ( $\left.\Psi-1\right)$ for odd values of $i$, and differentiable of type ( $\Psi-2)$ for other values, and an ith element of $\psi^{j \Psi}(v)$ is differentiable of type ( $(\Psi-1)$ for odd values of $j$. Then $\Phi(v) \ominus \psi(v)$ is differentiable of order $p \Psi$ if $\mathrm{H}$-difference $\Phi^{p \Psi}(v) \ominus \psi^{p \Psi}(v)$ exists, and we have

$$
(\Phi \ominus \psi)^{p^{\Psi}}(v)=\Phi^{p^{\Psi}}(v)+(-1) \psi^{p \Psi}(v) .
$$

2. If a kth element of $\Phi^{i \Psi}(v)$ is differentiable of type ( $\left.\Psi-1\right)$ for even values of $i$, and an ith element of $\psi^{j \Psi}(v)$ is differentiable of type $(\Psi-1)$ for odd values of $j$. Then $\Phi(v) \ominus \psi(v)$ is differentiable of order $p \Psi$ if H-difference $\Phi^{p^{\Psi}}(v) \ominus \psi^{p \Psi}(v)$ exists, and we have

$$
(\Phi \ominus \psi)^{p^{\Psi}}(v)=(-1) \Phi^{p^{\Psi}}(v)+\psi^{p \Psi}(v) .
$$

3. If a kth element of $\Phi^{i \Psi}(v)$, and an ith element of $\psi^{j \Psi}(v)$ is differentiable of type ( $\Psi$ 1 ) for even values of $i$, and $j$ respectively, and differentiable of type ( $\Psi-2)$ for other values. Then $\Phi(v) \ominus \psi(v)$ is differentiable of order $p \Psi$ if H-difference $\Phi^{p \Psi}(v) \odot$ $\psi^{\mathrm{p \Psi}}(v)$ exists, and we have

$$
(\Phi \odot \psi)^{p^{\Psi}}(v)=\psi^{p \Psi}(v) \odot(-1) \Phi^{p \Psi}(v) .
$$

4. If a kth element of $\Phi^{i \Psi}(v)$, and an ith element of $\psi^{j \psi}(v)$ is differentiable of type $(\Psi-1)$ for odd values of $i$, and $j$, and differentiable of type ( $\Psi-2)$ for other values. Then $\Phi(v) \ominus \psi(v)$ is differentiable of order $p \Psi$ if H-difference $\Phi^{p^{\Psi}}(v) \ominus \psi^{p \Psi}(v)$ exists, and we have

$$
(\Phi \ominus \psi)^{p^{\Psi}}(v)=\Phi^{p \Psi}(v) \ominus \psi^{p \Psi}(v) .
$$

Example 5.8. Define fuzzy-valued functions $\Phi$ and $\psi$ with $\gamma$-cuts

$$
\Phi(v)=[3+\eta, 5-\gamma] \cdot \sin v .
$$

Here

$$
\psi(v)=[4+\gamma, 6-\gamma] \cdot\left(v^{\frac{3}{2}}-v+v^{\frac{1}{2}}-1\right),
$$

and $v$ ranges from 0 to $\frac{\pi}{2}$. 
1. If fuzzy-valued functions $\Phi$ and it's first fuzzy conformable derivative are differentiable of type ( $\Psi-1)$, and $\psi$, and it's derivatives of order $\Psi$, and $2 \Psi$ are differentiable of type ( $\Psi-2)$ or if one of $\Phi$, and it's derivatives of order $\Psi$, and $2 \Psi$ are differentiable of type ( $\Psi-1)$, and other two are differentiable of type ( $\Psi-2)$, and one of $\psi$, and it's derivatives of order $\Psi$, and $2 \Psi$ are differentiable of type $(\Psi-2)$, and other two are differentiable of type ( $\Psi-1)$. Now by applying the above theorem, $\Phi(v) \ominus \psi(v)$ is differentiable of order $3 \Psi$ if H-differences $\Phi^{\Psi}(v) \ominus \psi^{\Psi}(v), \Phi^{2 \Psi}(v) \ominus \psi^{2 \Psi}(v)$ exist, and we have

$$
(\Phi \ominus \psi)^{3 \Psi}(v)=(-\cos v) \cdot[3+\gamma, 5-\gamma]+(-1)[4+\gamma, 6-\gamma] \cdot\left(\frac{-3}{8}\left(1-v^{\frac{-1}{2}}\right)\right) .
$$

2. If fuzzy-valued functions $\Phi$ it's derivatives of order $\Psi$, and $2 \Psi$ are differentiable of type $(\Psi-2)$, and $\psi$, and it's derivatives of order $\Psi$, and $2 \Psi$ are differentiable of type ( $\Psi-1)$ or if one of $\Phi$, and derivatives of order $\Psi$, and $2 \Psi$ are differentiable of type ( $\Psi-2)$, and other two are differentiable of type ( $\Psi-1)$, and one of $\psi$, and it's derivatives of order $\Psi$, and $2 \Psi$ are differentiable of type ( $\Psi-1)$, and the other two are differentiable of type ( $\Psi-2)$. Now by applying the above theorem, $\Phi(v) \ominus \psi(v)$ is differentiable of order $3 \Psi$ if H-differences $\Phi^{\Psi}(v) \ominus \psi^{\Psi}(v), \Phi^{2 \Psi}(v) \ominus \psi^{2 \Psi}(v)$ exist, and we have

$$
(\Phi \ominus \psi)^{3 \Psi}(v)=(-1)[3+\gamma, 5-\gamma] \cdot(-\cos v)+[4+\gamma, 6-\gamma] \cdot\left(\frac{-3}{8}\left(1-v^{\frac{-1}{2}}\right)\right) .
$$

3. If one of $\Phi$, and it's derivatives of order $\Psi$, and $2 \Psi$ are differentiable of type $(\Psi-2)$, and other two are differentiable of type $(\Psi-1)$, and one of $\Psi$, and it's derivatives of order $\Psi$, and $2 \Psi$ are differentiable of type ( $\Psi-2)$. Now by applying the above theorem, $\Phi(v) \ominus \psi(v)$ is differentiable of order $3 \Psi$ if H-differences $\Phi^{\Psi}(v) \ominus \psi^{\Psi}(v)$, $\Phi^{2 \Psi}(v) \ominus \psi^{2 \Psi}(v)$ exist, and we have

$$
(\Phi \ominus \psi)^{3 \Psi}(v)=[4+\gamma, 6-\gamma] \cdot\left(\frac{-3}{8}\left(1-v^{\frac{-1}{2}}\right)\right) \ominus(-1)[3+\gamma, 5-\gamma] \cdot(-\cos v) .
$$

4. If one of $\Phi$, and it's derivatives of order $\Psi$, and $2 \Psi$ are differentiable of type ( $\Psi-1)$, and the other two are differentiable of type ( $\Psi-2)$, and all of $\psi$, and it's derivatives of order $\Psi$, and $2 \Psi$ are differentiable of type ( $\Psi-1)$. Now by applying the above theorem, $\Phi(v) \ominus \psi(v)$ is differentiable of order $3 \Psi$ if H-differences $\Phi^{\Psi}(v) \ominus \psi^{\Psi}(v)$, $\Phi^{2 \Psi}(v) \ominus \psi^{2 \Psi}(v)$ exist, and we have

$$
(\Phi \ominus \psi)^{3 \Psi}(v)=[3+\gamma, 5-\gamma] \cdot(-\cos v) \ominus[4+\gamma, 6-\gamma] \cdot\left(\frac{-3}{8}\left(1-v^{\frac{-1}{2}}\right)\right) .
$$

Theorem 5.9. If $\mathrm{p}$ is odd, then we have four cases. 
1 If a kth element of $\Phi^{i \Psi}(v)$ is differentiable of type ( $\left.\Psi-1\right)$ for odd values of $i$, and differentiable of type ( $\Psi-2)$ for other values, and an ith element of $\psi^{j \Psi}(v)$ is differentiable of type $(\Psi-1)$ for odd values of $j$. Then $\Phi(v)+\psi(v)$ is differentiable of order $\mathrm{p} \Psi$, and we have

$$
(\Phi(v)+\psi(v))^{\mathrm{p} \Psi}=\Phi^{\mathrm{p} \Psi}(v)+\psi^{\mathrm{p} \Psi}(v) .
$$

2. If a kth element of $\Phi^{i \Psi}(v)$ is differentiable of type ( $\left.\Psi-1\right)$ for even values of $i$, and differentiable of type ( $\Psi-2)$ for other values, and an ith element of $\psi^{j \Psi}(v)$ is differentiable of type ( $\Psi-1)$ for odd values of $j$. Then $\Phi(v)+\psi(v)$ is differentiable of order $p \Psi$, and we have

$$
(\Phi(v)+\psi(v))^{p^{\Psi}}=(-1) \Phi^{p^{\Psi}}(v)+(-1) \psi^{p \Psi}(v) .
$$

3. If a kth element of $\Phi^{i \Psi}(v)$ is differentiable of type ( $\left.\Psi-1\right)$ for even values of $j$, and differentiable of type ( $\Psi-2)$ for other values, and an ith element of $\psi^{j \Psi}(v)$ is differentiable of type ( $\Psi-1)$ for even values of $j$. Then $\Phi(v)+\psi(v)$ is differentiable of order $p \Psi$, and we have

$$
(\Phi(v)+\psi(v))^{\mathrm{p} \Psi}=(-1) \Phi^{\mathrm{p} \Psi}(v) \odot \psi^{\mathrm{p} \Psi}(v) .
$$

4. If a kth element of $\Phi^{i \Psi}(v)$ is differentiable of type ( $\left.\Psi-1\right)$ for odd values of $i$, and differentiable of type ( $\Psi-2)$ for other values, and an ith element of $\psi^{j \Psi}(\nu)$ is differentiable of type ( $\Psi-1)$ for odd values of $j$. Then $\Phi(v)+\psi(v)$ is differentiable of order $\mathrm{p} \Psi$, and we have

$$
(\Phi(v)+\psi(v))^{p \Psi}=\psi^{p \Psi}(v) \odot(-1) \Phi^{p \Psi}(v) .
$$

Theorem 5.10. If $\mathrm{p}$ is even, then we have four possibilities.

1. If a kth element of $\Phi^{i \Psi}(v)$ is differentiable of type ( $\left.\Psi-1\right)$ for odd values of $i$, and differentiable of type $(\Psi-2)$ for other values, and an ith element of $\psi^{j \Psi}(v)$ is differentiable of type ( $\Psi-1)$ for odd values of $j$, and differentiable of type $(\Psi-2)$ for other values. Then $\Phi(v) \ominus \psi(v)$ is differentiable of order $p \Psi$ if H-difference $\Phi^{\mathrm{p} \Psi}(v) \ominus \psi^{\mathrm{p} \Psi}(v)$ exists, and we have

$$
(\Phi \ominus \psi)^{p^{\Psi}}(v)=\Phi^{p^{\Psi}}(v) \ominus \psi^{p \Psi}(v) .
$$

2. If a kth element of $\Phi^{i \Psi}(v)$ is differentiable of type ( $\left.\Psi-1\right)$ for even values of $i$, and differentiable of type ( $\Psi-2)$ for other values, and an ith element of $\psi^{j} \Psi(v)$ is differentiable of type ( $\Psi-1)$ for odd values of $j$, and differentiable of type $(\Psi-2)$ for other values. Then $\Phi(v) \ominus \psi(v)$ is differentiable of order $p \Psi$ if H-difference $\Phi^{\mathrm{p} \Psi}(v) \ominus \psi^{\mathrm{p}^{\Psi}}(v)$ exists, and we have

$$
(\Phi \ominus \psi)^{p^{\Psi}}(v)=(-1) \Phi^{p^{\Psi}}(v) \ominus \psi^{p \Psi}(v) .
$$


3. If a kth element of $\Phi^{i \Psi}(v)$ is differentiable of type ( $\left.\Psi-1\right)$ for odd values of $i$, and an ith element of $\psi^{j \Psi}(v)$ is differentiable of type ( $\left.\Psi-1\right)$ for even values of $j$. Then $\Phi(v) \ominus \psi(v)$ is differentiable of order $p \Psi$ if H-difference $\Phi^{p^{\Psi}}(v) \ominus \psi^{\mathrm{p} \Psi}(v)$ exists, and we have

$$
(\Phi \ominus \psi)^{p \Psi}(v)=\psi^{p \Psi}(v) \ominus(-1) \Phi^{p \Psi}(v) .
$$

4. If a kth element of $\Phi^{i \Psi}(v)$ is differentiable of type ( $\left.\Psi-1\right)$ for even values of $i$, and an ith element of $\psi^{j \Psi}(v)$ is differentiable of type ( $\left.\Psi-1\right)$ for odd values of $j$. Then $\Phi(v) \ominus \psi(v)$ is differentiable of order $p \Psi$ if H-difference $\Phi^{p^{\Psi}}(v) \ominus \psi^{p \Psi}(v)$ exists, and we have

$$
(\Phi \ominus \psi)^{p^{\Psi}}(v)=\Phi^{p^{\Psi}}(v)+(-1) \psi^{p \Psi}(v) .
$$

Example 5.11. Define fuzzy-valued functions $\Phi$ and $\psi$, where $\Phi(v)=[3+\gamma, 5-\gamma]$. $\sin v$, and $\psi(v)=[4+\gamma, 6-\gamma] \cdot\left(v^{\frac{3}{2}}-v+v^{\frac{1}{2}}-1\right)$, where $v$ ranges from 0 to $\frac{\pi}{2}$.

We have four possibilities here.

1. If fuzzy-valued functions $\Phi$ and $\psi$ and it's derivatives are $\Psi$-differentiable of the same type or if $\Phi$ and it's first three $\Psi$ - derivatives are differentiable of type ( $\Psi-1)$, and $\psi$ and it's first three $\Psi$-derivatives are differentiable of type $(\Psi-2)$ or vice versa. Then by applying the above theorem, $\Phi(v) \ominus \psi(v)$ is differentiable of order $4 \Psi$ if H-differences $\Phi^{\Psi}(v) \ominus \psi^{\Psi}(v), \Phi^{2 \Psi}(v) \ominus \psi^{2 \Psi}(v), \Phi^{(3 \Psi)}(v) \ominus \psi^{(3 \Psi)}(v)$ exist, and we have

$$
(\Phi \odot \psi)^{(4 \Psi)}(v)=(-\cos v) \cdot[3+\gamma, 5-\gamma] \ominus[4+\gamma, 6-\gamma] \cdot\left(\frac{-3}{16} \frac{1}{v}\right) .
$$

2. If one of $\Phi$ and it's first three $\Psi$-derivatives are differentiable of type ( $\Psi-2)$ and other two are differentiable of type ( $\Psi-1)$, and one of $\psi$, and it's first three $\Psi$-derivatives are differentiable of type ( $\Psi-1)$, and other two are differentiable of type ( $\Psi-2)$ or if one of $\Phi$, and it's first three $\Psi$-derivatives are differentiable of type ( $\Psi-1)$, and other three are differentiable of type ( $\Psi-2)$, and one of $\psi$, and it's first three $\Psi$-derivatives are differentiable of type ( $\Psi-1)$, and other three are differentiable of type ( $\Psi-2)$ or vice versa. Then by applying above theorem, $\Phi(v) \ominus \psi(v)$ is differentiable of order $4 \Psi$ if H-differences $\Phi^{\Psi}(v) \ominus \psi^{\Psi}(v), \Phi^{2 \Psi}(v) \ominus \psi^{2 \Psi}(v), \Phi^{(3 \Psi)}(v) \ominus \psi^{(3 \Psi)}(v)$ exist, and we have

$$
(\Phi \ominus \psi)^{(4 \Psi)}(v)=(-1)[3+\gamma, 5-\gamma] \cdot(-\cos v) \odot[4+\gamma, 6-\gamma] \cdot\left(\frac{-3}{16} \frac{1}{v}\right) .
$$

3. If one of $\Phi$ it's first three $\Psi$-derivatives are differentiable of type ( $\Psi-2)$, and other three are differentiable of type ( $\Psi-2)$, and all of $\psi$, and it's derivatives are differentiable of type ( $\Psi-2)$ or all are differentiable of type ( $\Psi-1)$. Then by applying above theorem, $\Phi(v) \ominus \psi(v)$ is differentiable of order $4 \Psi$ if H-differences $\Phi^{\psi}(v) \ominus \psi^{\Psi}(v)$, $\Phi^{2 \Psi}(v) \odot \psi^{2 \Psi}(v), \Phi^{(3 \Psi)}(v) \ominus \psi^{(3 \Psi)}(v)$ exist, and we have

$$
(\Phi \ominus \psi)^{(4 \Psi)}(v)=(-1)[3+\gamma, 5-\gamma] \cdot(-\cos v) \ominus(-1)[4+\gamma, 6-\gamma] \cdot\left(\frac{-3}{16} \frac{1}{v}\right) .
$$


4. If all of $\Phi$ and it's $\Psi$-derivatives are differentiable of type ( $\Psi-2)$, and one of $\psi$ and it's first three $\Psi$ - derivatives are differentiable of type ( $\Psi-1)$, and other two are fuzzy $\Psi$ - differentiable of type ( $\Psi-2)$ or if $\Phi$ and it's $\Psi$ - derivatives are differentiable of type ( $\Psi-1)$ and one of $\psi$ and it's three $\Psi$ - derivatives are differentiable of type $(\Psi-2)$, and other three are differentiable of type ( $\Psi-1)$. Then by applying above theorem, $\Phi(v) \ominus \psi(v)$ is differentiable of order $4 \Psi$ if H-differences $\Phi^{\Psi}(v) \odot \psi^{\Psi}(v)$, $\Phi^{2 \Psi}(v) \ominus \psi^{2 \Psi}(v), \Phi^{(3 \Psi)}(v) \ominus \psi^{(3 \Psi)}(v)$ exist, and we have

$$
(\Phi \ominus \psi)^{(4 \Psi)}(v)=[3+\gamma, 5-\gamma] \cdot(-\cos v)+(-1)[4+\gamma, 6-\gamma] \cdot\left(\frac{-3}{16} \frac{1}{v}\right) .
$$

\section{Conclusion}

Our choice has certain advantages concerning other techniques for solving the fuzzy conformable differential equations. First, solutions have a decreasing length of support. Second, it can also solve the fuzzy conformable partial differential equations. Third, a numerical solution of the fuzzy conformable differential equations can be obtained using this technique, which is not possible with the fuzzy differential inclusion. The solution obtained by using conformable strongly generalized differentiability is not unique. But it can be seen as an advantage because we can choose among those solutions, the best one that better reflects the real-life situation. The conformable strongly generalized derivative has certain advantages over other fuzzy fractional derivatives. For example, a solution of the fuzzy differential equations can easily be obtained using a conformable strongly generalized derivative. It possesses the fundamental properties of the fuzzy derivatives. For example, the derivative of a product of fuzzy-valued functions, fuzzy chain rule, fuzzy mean value theorem, fuzzy Roll's theorem, etc. In the case of other fractional derivatives, some functions are not infinitely differentiable at some point, so the fuzzy Taylor series does not exist for those fuzzy-valued functions, where using fuzzy conformable calculus, these functions are infinitely differentiable.

In the future, we aim to introduce the fuzzy conformable integral calculus, and, using these results, we will solve the fuzzy conformable differential equations of order $\Psi, 2 \Psi$, and $p \Psi$. This combination of two theories of fuzzy calculus and conformable calculus will help us to obtain better results in the modeling of real-life phenomena.

\section{References}

[1] Zadeh LA (1996). Fuzzy sets. In Fuzzy sets, fuzzy logic, and fuzzy systems, 6: 394-432. https://doi. org/10.1142/2895

[2] Mizumoto M, Tanaka K (1976). The four operations of arithmetic on fuzzy numbers. Syst. Comput. Controls, 7(5): 73-81.

[3] Dubois D, Prade H (1982). Towards fuzzy differential calculus part 3: Differentiation. Fuzzy sets and systems, 8(3): 225-233. https://doi.org/10.1016/S0165-0114(82)80001-8

[4] Chang SSL, Zadeh LA (1996). On fuzzy mapping and control. In Fuzzy sets, fuzzy logic, and fuzzy systems, 180-184. https://doi.org/10.1142/9789814261302_0012

[5] Dubois D, Prade H (1978). Operations on fuzzy numbers. International Journal of systems science, 9(6): 613-626. https://doi.org/10.1080/0020772780894172

[6] Hukuhara M (1967). Integration des applications mesurables dont la valeur est un compact convexe. Funkcialaj Ekvacioj, 10(3): 205-223. 
[7] Barnabás B, Imre Rudas J, Attila Bencsik L (2007), First order linear fuzzy differential equations under generalized differentiability. Information sciences, 177(7): 1648-1662. https://doi .org/10.1016/j . ins . 2006.08 .021

[8] Abu Aqrab O, Al-Smadi M, Momani S, Hayat T (2017). Application of reproducing kernel algorithm for solving second-order, two-point fuzzy boundary value problems. Soft Computing, 21(23): 7191-7206. https: //doi.org/10.1007/s00500-016-2262-3

[9] Abu Arqub O (2017), Adaptation of reproducing kernel algorithm for solving fuzzy Fredholm-Volterra integrodifferential equations. Neural Computing and Applications, 28(7): 1591-1610. https://doi.org/10. $1007 / \mathrm{s} 00521-015-2110-\mathrm{x}$

[10] Abu Arqub O, AL-Smadi M, Momani S, Hayat T (2016). Numerical solutions of fuzzy differential equations using reproducing kernel Hilbert space method. Soft Computing, 20(8): 3283-3302. https://doi.org/10. 1007/s00500-015-1707-4

[11] Bede B (2013). Mathematics of Fuzzy Sets and Fuzzy Logic. Studies in Fuzziness and Soft Computing, Springer, Berlin, Heidelberg. https://doi .org/10.1007/978-3-642-35221-8

[12] Abu Aqrab O, Singh J, Maayah B, Alhodaly M (2021). Reproducing kernel approach for numerical solutions of fuzzy fractional initial value problems under the Mittag-Leffler kernel differential operator. Mathematical Methods in the Applied Sciences, 2021: 1-22. https://doi.org/10.1002/mma.7305

[13] Abu Aqrab O, Singh J, Alhodaly M (2021). Adaptation of kernel functions-based approach with AtanganaBaleanu-Caputo distributed order derivative for solutions of fuzzy fractional Volterra and Fredholm integrodifferential equations. Mathematical Methods in the Applied Sciences, 2021: 1-28. https://doi.org/10. 1002/mma.7228

[14] Khalil R, Al Horani M, Yousef A, Sababheh M (2014). A new definition of fractional derivative. Journal of Computational, and Applied Mathematics, 264: 65-70. https://doi.org/10.1016/j.cam.2014.01. 002

[15] Abdeljawad T (2015). On conformable fractional calculus. Journal of Computational and Applied Mathematics, 279: 57-66. https://doi.org/10.1016/j.cam.2014.10.016

[16] Abdelhakim AA, Machado JAT (2019). A critical analysis of the conformable derivative. Nonlinear Dynamics, 95(4): 3063-3073. https: //doi .org/10.1142/S0218348X21500638

[17] Abdon A, Baleanu D, Alsaedi A (2015). New properties of conformable derivative. Open Mathematics, 13(1): 889-898. https://doi.org/10.1515/math-2015-0081

[18] Omid Birgani T, Chandok S, Dedović N, Radenović S (2019). A note on some recent results of the conformable fractional derivative. Advances in the Theory of Nonlinear Analysis and its Applications, 3(1): 11-17. https://doi.org/10.31197/atnaa. 482525

[19] Bohner M, Fuat Hatipoğlu V (2018). Dynamic cobweb model with conformable fractional derivatives. Mathematical Methods in the Applied Sciences, 41(18): 9010-9017. https://doi.org/10.1016/j.nahs. 2018.09.004

[20] Ma X, Wenqing W, Zeng B, Yong W, Xinxing W (2020). The conformable fractional grey system model. ISA transactions, 96: 255-271. https://doi.org/10.1016/j.isatra.2019.07.009

[21] Khalil R, Al Horani M, Abu Hammad M (2019). Geometric meaning of conformable derivative via fractional cords. Journal of Mathematics and Computer Science, 19: 241-245. http://dx.doi.org/10.22436/ jmcs.019.04.03

[22] Younus A, Abdeljwad T, Tazeen G (2020). On Stability Criteria of Fractal Differential Systems of Conformable Type. Fractals, 28(8): 1-9. https://doi .org/10.1142/S0218348X20400095

[23] Younas A, Abdeljawad T, Rida B, Anum Z, Manar Alqudah A (2020). Linear conformable differential system and its controllability. Advances in Difference Equations 2020, 449: 1-26. https://doi.org/10. 1186/s13662-020-02899-0

[24] Younus A, Khizra B, Tunç C (2020). Existence of resolvent for conformable fractional Volterra integral equations. Applications \& Applied Mathematics, 15(1): 372-39.

[25] Abu Aqrab O, Al-Smadi M (2020), fuzzy conformable fractional differential equations: Novel extended approach, and new numerical solutions. Soft Computing, 24: 12501-12522. https://doi.org/10.1007/ s00500-020-04687-0.

[26] Atefeh A, Allahviranloo T, Gouyandeh Z (2018). Some fundamental results on fuzzy calculus. Iranian Journal of Fuzzy Systems, 15(3): 27-46. 10.22111/IJFS. 2018.3948

[27] Gomes LT, Laécio CB, Barnabas B (2015). fuzzy differential equations in various approaches. Springer Briefs in Mathematics, Springer, Cham. https://doi.org/10.1007/978-3-319-22575-3

[28] Hooshangian L, Tofigh A (2014). A new method to find fuzzy nth order derivation, and applications to fuzzy 
$\mathrm{N}$ th order arithmetic based on generalized H-derivation. An International Journal of Optimization, and Control: Theories \& Applications, 4(2): 105-121. https ://doi.org/10.11121/ijocta.01.2014.00183

[29] Khastan A, Bahrami F, Ivaz (2009). New results on multiple solutions for nth-order fuzzy differential equations under generalized differentiability. Boundary value problems, Article ID 395714, 2009: 1-13. https://doi.org/10.1155/2009/395714 